\title{
A Corotational Finite Element Method Combined with Floating Frame Method for Large Steady-State Deformation and Free Vibration Analysis of a Rotating-Inclined Beam
}

\author{
Ming Hsu Tsai, ${ }^{1}$ Wen Yi Lin, ${ }^{2}$ Yu Chun Zhou, ${ }^{1}$ and Kuo Mo Hsiao ${ }^{1}$ \\ ${ }^{1}$ Department of Mechanical Engineering, National Chiao Tung University, Hsinchu 300, Taiwan \\ ${ }^{2}$ Department of Mechanical Engineering, De Lin Institute of Technology, Tucheng 236, Taiwan \\ Correspondence should be addressed to Kuo Mo Hsiao, kmhsiao@mail.nctu.edu.tw
}

Received 30 March 2011; Accepted 19 May 2011

Academic Editor: Delfim Soares Jr.

Copyright ( 2011 Ming Hsu Tsai et al. This is an open access article distributed under the Creative Commons Attribution License, which permits unrestricted use, distribution, and reproduction in any medium, provided the original work is properly cited.

\begin{abstract}
A corotational finite element method combined with floating frame method and a numerical procedure is proposed to investigate large steady-state deformation and infinitesimal-free vibration around the steady-state deformation of a rotating-inclined Euler beam at constant angular velocity. The element nodal forces are derived using the consistent second-order linearization of the nonlinear beam theory, the d'Alembert principle, and the virtual work principle in a current inertia element coordinates, which is coincident with a rotating element coordinate system constructed at the current configuration of the beam element. The governing equations for linear vibration are obtained by the first-order Taylor series expansion of the equation of motion at the position of steady-state deformation. Numerical examples are studied to demonstrate the accuracy and efficiency of the proposed method and to investigate the steady-state deformation and natural frequency of the rotating beam with different inclined angle, angular velocities, radius of the hub, and slenderness ratios.
\end{abstract}

\section{Introduction}

Rotating beams are often used as a simple model for propellers, turbine blades, and satellite booms. Rotating beam differs from a nonrotating beam in having additional centrifugal force and Coriolis effects on its dynamics. The vibration analysis of rotating beams has been extensively studied [1-25]. However, the vibration analysis of rotating beam with inclination angle, which is considered in the recent computer cooling fan design on the natural frequencies of rotating beams [21], is rather rare in the literature [10, 19, 21, 22]. 


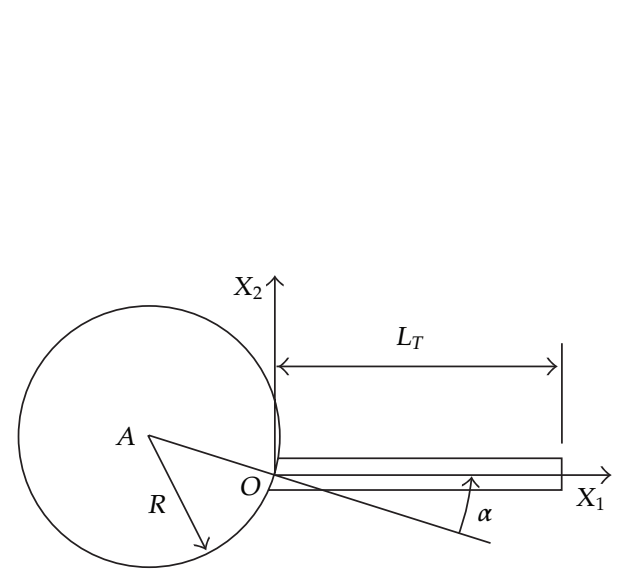

(a)

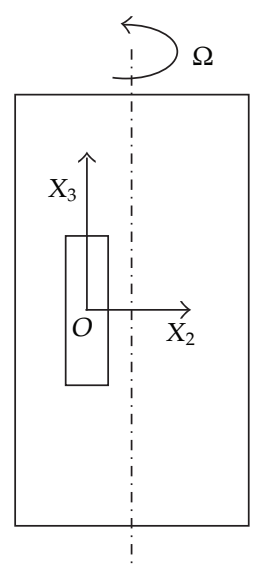

(b)

Figure 1: A rotating inclined beam, (a) top view, (b) side view.

In [21, 22], the effect of the steady-state axial deformation and the inclination angle on the natural frequencies of the rotating beam was investigated. However, the lateral steadystate deformation and its effects on the natural frequencies of the rotating beam were not considered in $[21,22]$. To the authors' knowledge, the lateral steady-state deformation and its effects on the lagwise bending and axial vibration of rotating inclined beams are not reported in the literature.

It is well known that the spinning elastic bodies sustain a steady-state deformation (time-independent deformation) induced by constant rotation [26]. For rotating beams with an inclination angle as shown in Figure 1, the steady-state deformations include axial deformation and lateral deformation. The linear solution of the steady-state deformation of rotating-inclined beam induced by constant rotation can be easily obtained using mechanics of materials. However, the centrifugal stiffening effect on the steady lateral deformation is significant for slender rotating-inclined beam, and the centrifugal force is configuration dependent load; thus the linear solution of the steady-state deformation of rotating inclined beam may be not accurate enough. The lagwise bending and axial vibration of rotating inclined beams are coupled due to the Coriolis effects $[15,24]$ and the lateral steady-state deformation. The accuracy of the frequencies obtained from linearizing about the steadystate deformation is dependent on the accuracy of the steady-state deformation and the accuracy of the linearized perturbation [6,12]. Thus, the geometrical nonlinearities that arise due to steady-state deformation should be considered. In [6], the rotating beam with pretwist, precone, and setting angle is studied. The undeformed state of the rotating beam is chosen to be the reference state to define the deformation parameters of the rotating beam. The geometric nonlinearities up to the second degree are considered. The Galerkin method, with vibration modes of nonrotating beam, is employed for the solution of both steadystate nonlinear equations and linear perturbation equations. In [8], it is reported that for a cantilever beam with a tip mass, even up to the third degree geometric nonlinearities are considered, in some cases, very inaccurate eigenvalues for the perturbed linearized equation of motion are obtained. The formulation used in $[6,8,12]$ may be regarded as a total Lagrangian (TL) formulation combined with the floating frame method. In order to capture correctly all inertia effects and coupling among bending, twisting, and stretching 
deformations of the rotating beam, the governing equations of the rotating beam might be derived by the fully geometrically nonlinear beam theory $[12,27,28]$. The exact expressions for the inertia, deformation forces, and the governing equations of the rotating beam, which are required in a TL formulation for large displacement/small strain problems, are highly nonlinear functions of deformation parameters. However, the dominant factors in the geometrical nonlinearities of beam structures are attributable to finite rotations, with the strains remaining small. For a beam structures discretized by finite elements, this implies that the motion of the individual elements to a large extent will consist of rigid body motion. If the rigid body motion part is eliminated from the total displacements and the element size is properly chosen, the deformational part of the motion is always small relative to the local element axes; thus in conjunction with the corotational formulation, the higherorder terms of nodal deformation parameters in the element deformation and inertia nodal forces may be neglected by consistent linearization [28, 29]. In [29], Hsiao et al. presented a corotational finite element formulation and numerical procedure for the dynamic analysis of planar beam structures. Both the element deformation and inertia forces are systematically derived by consistent linearization of the fully geometrically nonlinear beam theory using the $d^{\prime}$ Alembert principle and the virtual work principle. This formulation and numerical procedure were proven to be very effective by numerical examples studied in [29]. However, because the nodal displacements and rotations, velocities, accelerations, and the equations of motion of the system are defined in terms of a fixed global coordinate system, the formulation proposed in [29] cannot be used for steady-state deformation and free vibration analysis of a rotating-inclined beam. The absolute nodal coordinate formulation [30,31] is used to large rotation and large deformation problems. Numerical results show that the absolute nodal coordinate formulation can be effectively used in the large deformation problems. However, the mass matrix of the finite elements in $[30,31]$ is a constant matrix, and therefore, the centrifugal and Coriolis forces are equal to zero. Thus, the absolute nodal coordinate formulation cannot be used for steady-state deformation and free vibration analysis of a rotating inclined beam.

The objective of this study is to present a corotational finite element method combined with floating frame method and a numerical procedure for large steady-state deformation and free vibration analysis of a rotating-inclined beam at constant angular velocity. The nodal coordinates, displacements and rotations, absolute velocities, absolute accelerations, and the equations of motion of the system are defined in terms of an inertia global coordinate system which is coincident with a rotating global coordinate system rigidly tied to the rotating hub, while the total deformations in the beam element are measured in an inertia element coordinate system which is coincident with a rotating element coordinate system constructed at the current configuration of the beam element. The rotating element coordinates rotate about the hub axis at the angular speed of the hub. The inertia nodal forces and deformation nodal forces of the beam element are systematically derived by the virtual work principle, the d'Alembert principle, and consistent second-order linearization of the fully geometrically nonlinear beam theory [27-29] in the element coordinates. Due to the consideration of the exact kinematics of Euler beam, some coupling terms of axial and flexural deformations are retained in the element internal nodal forces. The element equations are constructed first in the inertia element coordinate system and then transformed to the inertia global coordinate system using standard procedure.

The infinitesimal-free vibrations of rotating beam are measured from the position of the corresponding steady-state deformation. The governing equations for linear vibration of 
rotating beam are obtained by the first-order Taylor series expansion of the equation of motion at the position of steady-state deformation.

Dimensionless numerical examples are studied to demonstrate the accuracy and efficiency of the proposed method and to investigate the effect of inclination angle and slenderness ratio on the steady-state deformation and the natural frequency for rotating inclined Euler beams at different angular speeds.

\section{Formulation}

\subsection{Description of Problem}

Consider an inclined uniform Euler beam of length $L_{T}$ rigidly mounted with an inclination angle $\alpha$ on the periphery of rigid hub with radius $R$ rotating about its axis fixed in space at a constant angular speed $\Omega$ as shown in Figure 1. The axis of the rotating hub is perpendicular to one of the principal directions of the cross section of the beam. The deformation displacements of the beam are defined in an inertia rectangular Cartesian coordinate system which is coincident with a rotating rectangular Cartesian coordinate system rigidly tied to the hub.

Here only axial and lagwise bending vibrations are considered. It is well known that the beam sustains a steady-state deformations (time-independent deformation displacements) induced by constant rotation [26]. In this study, large displacement and rotation with small strain are considered in the steady-state deformation. The vibration (time-dependent deformation displacements) of the beam is measured from the position of the steady-state deformation, and only infinitesimal-free vibration is considered. Note that the axial and lagwise vibrations, which are coupled due to the Coriolis effects and the lateral steady-state deformation, cannot be analyzed independently. Here the engineering strain and stress are used for the measure of the strain and stress.

\subsection{Basic Assumptions}

The following assumptions are made in derivation of the beam element behavior.

(1) The beam is prismatic and slender, and the Euler-Bernoulli hypothesis is valid.

(2) The unit extension of the centroid axis of the beam element is uniform.

(3) The deformation displacements and rotations of the beam element are small.

(4) The strains of the beam element are small.

In conjunction with the corotational formulation and rotating frame method, the third assumption can always be satisfied if the element size is properly chosen. Thus, only the terms up to the second order of deformation parameters and their spatial derivatives are retained in element position vector, strain, and deformation nodal forces by consistent second-order linearization in this study.

\subsection{Coordinate Systems}

In order to describe the system, we define three sets of right-handed rectangular Cartesian coordinate systems. 


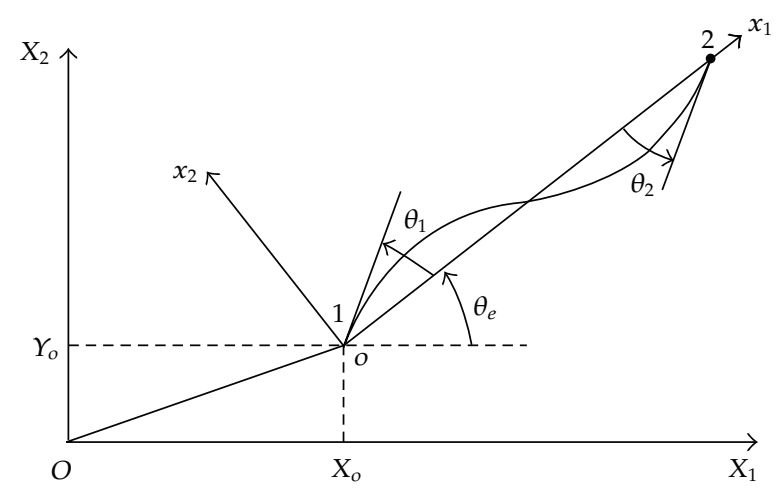

Figure 2: Coordinate systems.

(1) A rotating global set of coordinates, $X_{i}(i=1,2,3)$ (see Figures 1 and 2); the coordinates rotate about the hub axis at a constant angular speed $\Omega$ as shown in Figure 1. The origin of this coordinate system is chosen to be the intersection of the centroid axes of the hub and the undeformed beam. The $X_{1}$ axis is chosen to coincide with the centroid axis of the undeformed beam, and the $X_{2}$ and $X_{3}$ axes are chosen to be the principal directions of the cross section of the beam at the undeformed state. The direction of the axis of the rotating hub is parallel to the $X_{3}$ axis. The nodal coordinates, nodal deformation displacements, absolute nodal velocity, absolute nodal acceleration, and equations of motion of the system are defined in terms of an inertia global coordinate system which is coincident with the rotating global coordinate system.

(2) Element coordinates; $x_{i}(i=1,2,3)$ (see Figure 2), a set of element coordinates is associated with each element, which is constructed at the current configuration of the beam element. The coordinates rotate about the hub axis at a constant angular speed $\Omega$. The origin of this coordinate system is located at the element node 1 , the centroid of the end section. The $x_{1}$ axis is chosen to pass through two end nodes of the element; the directions of the $x_{2}$ and $x_{3}$ axes are chosen to coincide with the principal direction of the cross section in the undeformed state. Because only the displacements in $X_{1} X_{2}$ plane are considered, the directions of $x_{3}$ axis and $X_{3}$ axis are coincident. The position vector, deformations, absolute velocity, absolute acceleration, internal nodal forces, stiffness matrices, and inertia matrices of the elements are defined in terms of an inertia element coordinate system which is coincident with the rotating element coordinate system.

In this study, the direction of the axis of the rotating hub is parallel to the $X_{3}$ axis and only the displacements in $X_{1} X_{2}$ plane are considered. Thus, the angular velocity of the hub referred to the global coordinates may be given by

$$
\Omega_{G}=\left\{\begin{array}{lll}
0 & 0 & \Omega
\end{array}\right\},
$$

where the symbol \{\} denotes a column matrix, which is used through the paper. 


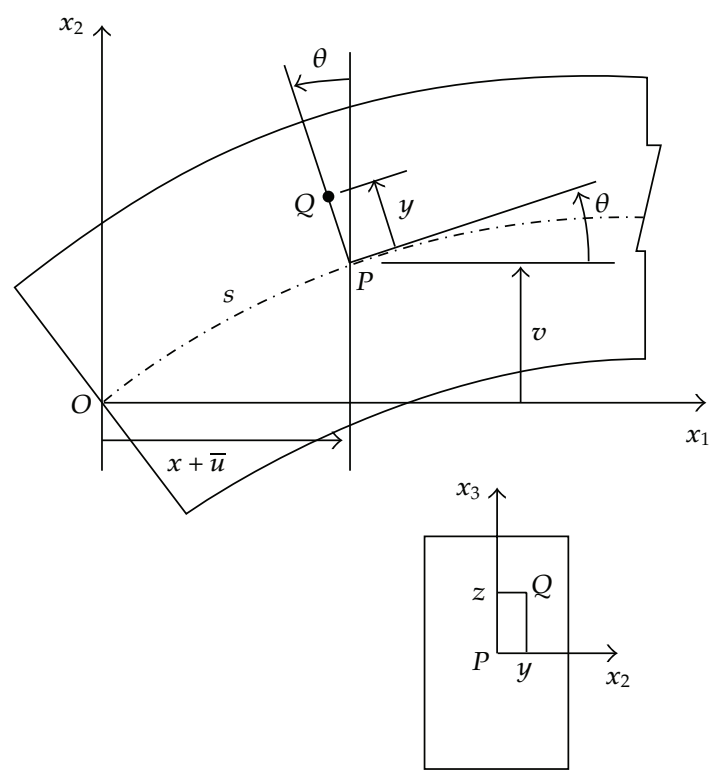

Figure 3: Kinematics of Euler beam.

\subsection{Kinematics of Beam Element}

Let $Q$ (Figure 3) be an arbitrary point in the beam element and $P$ the point corresponding to $Q$ on the centroid axis. The position vector of point $Q$ in the undeformed configurations referred to the current element coordinate system may be expressed as

$$
\mathbf{r}_{0}=\{x, y, z\}
$$

Using the approximation $\cos \theta \approx 1-(1 / 2) \theta^{2}, \sin \theta \approx \theta$, and $\left(1+\varepsilon_{c}\right) \approx 1$, retaining all terms up to the second order, the position vector of point $Q$ in the deformed configurations referred to the current element coordinate system may be expressed as

$$
\begin{gathered}
r=\left\{r_{1}, r_{2}, r_{3}\right\}=\left\{x_{p}-y \theta, y\left(1-\frac{1}{2} \theta^{2}\right)+v, z\right\}, \\
\theta \approx \sin \theta=\frac{\partial v(x, t)}{\partial s}=\frac{\partial v(x, t)}{\partial x} \frac{\partial x}{\partial s}=\frac{v^{\prime}}{1+\varepsilon_{c}} \approx v^{\prime}, \\
\varepsilon_{c}=\frac{\partial s}{\partial x}-1,
\end{gathered}
$$

where $x_{p}(x, t)$ and $v(x, t)$ are the $x_{1}$ and $x_{2}$ coordinates of point $P$, respectively, in the deformed configuration, $t$ is time, $\theta=\theta(x, t)$ is the angle counterclockwise measured from $x_{1}$ axis to the tangent of the centroid axis of the deformed beam, $\varepsilon_{c}$ is the unit extension of the centroid axis, and $s$ is the arc length of the deformed centroid axis measured from node 1 to point $P$. In this paper, ()$^{\prime}$ denotes ()$_{, x}=\partial() / \partial x$. 
Here, the lateral deflection of the centroid axis, $v(x, t)$, is assumed to be the Hermitian polynomials of $x$ and may be expressed by

$$
v(x, t)=\left\{N_{1}, N_{2}, N_{3}, N_{4}\right\}^{t}\left\{v_{1}, v_{1}^{\prime}, v_{2}, v_{2}^{\prime}\right\}=\mathbf{N}_{b}^{t} \mathbf{u}_{b},
$$

where $v_{j}=v_{j}(t)$ and $v_{j}^{\prime}=v_{j}^{\prime}(t)(j=1,2)$ are nodal values of $v$ and $v_{, x}$, respectively, at nodes $j$. Note that, due to the definition of the element coordinates, the values of $v_{j}(j=1,2)$ are zero. However, their variations and time derivatives are not zero. $N_{i}(i=1-4)$ are shape functions and are given by

$$
\begin{gathered}
N_{1}=\frac{1}{4}(1-\xi)^{2}(2+\xi), \quad N_{2}=\frac{L}{8}\left(1-\xi^{2}\right)(1-\xi), \\
N_{3}=\frac{1}{4}(1+\xi)^{2}(2-\xi), \quad N_{4}=\frac{L}{8}\left(-1+\xi^{2}\right)(1+\xi), \\
\xi=-1+\frac{2 x}{L},
\end{gathered}
$$

where $L$ is the length of the undeformed beam element.

Making use of assumptions $v_{, x} \ll 1$ and $\varepsilon_{c} \ll 1$, the relationship between $x_{p}(x, t)$, $v(x, t)$, and $x$ in (2.3) may be approximated by

$$
x_{p}(x, t)=u_{1}+\int_{0}^{x}\left(1+\varepsilon_{c}-\frac{1}{2} v_{, x}^{2}\right) d x,
$$

where $u_{1}$ is the displacement of node 1 in the $x_{1}$ direction. Note that due to the definition of the element coordinate system, the value of $u_{1}$ is equal to zero. However, the variation and time derivatives of $u_{1}$ are not zero.

The axial displacements of the centroid axis may be determined from the lateral deflections and the unit extension of the centroid axis using (2.9).

From (2.9), one may obtain

$$
\ell=L+u_{2}-u_{1}=x_{c}(L, t)-x_{c}(0, t)=\int_{0}^{L}\left(1+\varepsilon_{c}-\frac{1}{2} v_{, x}^{2}\right) d x
$$

in which $\ell$ is the current chord length of the centroid axis of the beam element and $u_{2}$ is the displacement of node 2 in the $x_{1}$ direction. Using the assumption of uniform extension of the centroid axis and (2.10), $\varepsilon_{c}$ in (2.10) maybe expressed by

$$
\begin{gathered}
\varepsilon_{c}=\frac{1}{L}\left(\mathbf{G}_{a}^{t} \mathbf{u}_{a}+\frac{1}{2} \mathbf{G}_{b}^{t} \mathbf{u}_{b}\right), \\
\mathbf{G}_{a}=\{-1,1\}, \\
\mathbf{u}_{a}=\left\{u_{1}, u_{2}\right\}, \\
\mathbf{G}_{b}=\int_{0}^{\mathbf{L}} \mathbf{N}_{b}^{\prime} v_{, x} d x .
\end{gathered}
$$


Substituting (2.11) into (2.9), one may obtain

$$
\begin{gathered}
x_{p}(x, t)=\mathbf{N}_{a}^{t} \mathbf{u}_{a}+x+\frac{\mathbf{x}}{2 L} \mathbf{G}_{b}^{t} \mathbf{u}_{b}-\frac{1}{2} \int_{0}^{x} v_{, x}^{2} d x, \\
\mathbf{N}_{a}=\left\{\frac{1-\xi}{2}, \frac{1+\xi}{2}\right\} .
\end{gathered}
$$

From (2.3) and the definition of engineering strain [32, 33], making use of the assumption of small strain, and retaining the terms up to the second order of deformation parameters, the engineering strain in the Euler beam may be approximated by

$$
\varepsilon_{11}=\varepsilon_{c}-y v^{\prime \prime}
$$

The absolute velocity and acceleration vectors of point $Q$ in the beam element may be expressed as

$$
\begin{gathered}
\mathbf{v}=\left\{v_{1}, v_{2}, v_{3}\right\}=\mathbf{v}_{o}+\boldsymbol{\Omega} \times \mathbf{r}+\dot{\mathbf{r}}, \\
\mathbf{a}=\left\{a_{1}, a_{2}, a_{3}\right\}=\mathbf{a}_{o}+\mathbf{\Omega} \times \mathbf{r}+\boldsymbol{\Omega} \times(\boldsymbol{\Omega} \times \mathbf{r})+\mathbf{\Omega} \times \dot{\mathbf{r}}+\ddot{\mathbf{r}}, \\
\mathbf{v}_{o}=\boldsymbol{\Omega} \times \mathbf{r}_{A o}, \\
\mathbf{a}_{o}=\left\{a_{o 1}, a_{o 2}, a_{o 3}\right\}=\boldsymbol{\Omega} \times\left(\boldsymbol{\Omega} \times \mathbf{r}_{A o}\right), \\
\boldsymbol{\Omega}=\mathbf{A}_{G E}^{t} \boldsymbol{\Omega}_{G}, \\
\mathbf{r}_{A o}=\mathbf{A}_{G E}^{t} \mathbf{r}_{A o G}, \\
\mathbf{r}_{A o G}=\mathbf{r}_{A O}+\mathbf{r}_{O o G}=\left\{R \cos \alpha+X_{o},-R \sin \alpha+Y_{o}, 0\right\},
\end{gathered}
$$

where $\mathbf{r}$ is the position vector of point $Q$ given in (2.3) referred to the current moving element coordinate system, the symbol ( ) denotes time derivative, $\Omega$ is the vector of angular velocity referred to the current inertia element coordinates, $\boldsymbol{\Omega}_{G}$ is the angular velocity of the hub referred to the global coordinates given in (2.1), $\mathbf{A}_{G E}$ is the transformation matrix between the current global coordinates and the current element coordinates, $\mathbf{v}_{o}$ and $\mathbf{a}_{o}$ are the absolute velocity and absolute acceleration of point $o$, the origin of the current element coordinates, $X_{O}$ and $Y_{o}$ are coordinates of point $o$ referred to the current global coordinates, $R$ is the radius of the hub, and $\alpha$ is inclination angle of the rotating beam. $\boldsymbol{\Omega} \times(\boldsymbol{\Omega} \times \mathbf{r})$ and $2 \boldsymbol{\Omega} \times \mathbf{r}$ are centripetal acceleration and Coriolis acceleration, respectively. $\dot{\mathbf{r}}$ and $\ddot{\mathbf{r}}$ are the velocity and acceleration of point $Q$ relative to the current moving element coordinates. From (2.3), (2.11) and (2.12), ir and $\ddot{r}$ may be expressed as

$$
\begin{aligned}
& \dot{\mathbf{r}}=\left\{\dot{r}_{1}, \dot{r}_{2}, \dot{r}_{3}\right\}=\left\{\dot{x}_{p}-y \dot{v}, x, \dot{v}-y \dot{v}_{, x} v, x, 0\right\}, \\
& \ddot{\mathbf{r}}=\left\{\ddot{r}_{1}, \ddot{r}_{2}, \ddot{r}_{3}\right\}=\left\{\ddot{x}_{p}-y \ddot{v}_{, x}, \ddot{v}-y \dot{v}_{, x}^{2}-y \ddot{v}_{, x} v_{, x}, 0\right\}, \\
& \dot{x}_{p}=\mathbf{N}_{a}^{t} \dot{\mathbf{u}}_{a}+\frac{x}{L} \mathbf{G}_{b}^{t} \dot{\mathbf{u}}_{b}-\int_{0}^{x} v_{, x} \dot{v}_{, x} d x,
\end{aligned}
$$




$$
\begin{aligned}
& \dot{\varepsilon}_{c}=\frac{1}{L}\left(\mathbf{G}_{a}^{t} \dot{\mathbf{u}}_{a}+\mathbf{G}_{b}^{t} \dot{\mathbf{u}}_{b}\right), \\
& \ddot{x}_{p}=\mathbf{N}_{a}^{t} \ddot{\mathbf{u}}_{a}+\frac{x}{L}\left(\mathbf{G}_{b}^{t} \ddot{\mathbf{u}}_{b}+\dot{\mathbf{G}}_{b}^{t} \dot{\mathbf{u}}_{b}\right)-\int_{0}^{x}\left(v_{, x} \ddot{v}_{, x}+\dot{v}_{, x} \dot{v}_{, x}\right) d x, \\
& \ddot{\varepsilon}_{c}=\frac{1}{L}\left(\mathbf{G}_{a}^{t} \ddot{\mathbf{u}}_{a}+\dot{\mathbf{G}}_{b}^{t} \dot{\mathbf{u}}_{b}+\mathbf{G}_{b}^{t} \ddot{\mathbf{u}}_{b}\right) .
\end{aligned}
$$

Note that the current element coordinates constructed at the current configuration of the beam element rotate about the hub axis at the angular velocity of the hub. Thus, the centripetal acceleration and Coriolis acceleration corresponding to the inertia forces of the rotating beam are unique. For nonrotating beam, $\boldsymbol{\Omega}=\mathbf{0}$ and $\dot{\mathbf{r}}$ and $\ddot{\mathbf{r}}$ are the absolute velocity and acceleration referred to the current element coordinate.

\subsection{Element Nodal Force Vector}

Let $u_{j}, \delta v_{j}$, and $\delta v_{j}^{\prime}(j=1,2)$ denote the virtual displacements in the $x_{1}$ and $x_{2}$ directions of the current inertia element coordinates, and virtual rotations applied at the element nodes $j$. The element nodal force corresponding to virtual nodal displacements $\delta u_{j}, \delta v_{j}$, and $\delta v_{j}^{\prime}(j=1,2)$ are $f_{i j}$, the forces in the $x_{i}(i=1,2)$ directions, and $m_{j}$ moments about the $x_{3}$ axis, at element local nodes $j$.

The element nodal force vector is obtained from the d'Alembert principle and the virtual work principle in the current inertia element coordinates. The virtual work principle requires that

$$
\begin{gathered}
\delta \mathbf{u}_{a}^{t} \mathbf{f}_{a}+\delta \mathbf{u}_{b}^{t} \mathbf{f}_{b}=\int_{V}\left(\delta \varepsilon_{11} \sigma_{11}+\rho \delta \mathbf{r}^{t} \mathbf{a}\right) d V, \\
\delta \mathbf{u}_{a}=\left\{\delta u_{1}, \delta u_{2}\right\}, \\
\delta \mathbf{u}_{b}=\left\{\delta v_{1}, \delta v_{1}^{\prime}, \delta v_{2}, \delta v_{2}^{\prime}\right\}, \\
\mathbf{f}_{a}=\mathbf{f}_{a}^{D}+\mathbf{f}_{a}^{I}=\left\{f_{11}, f_{12}\right\}, \\
\mathbf{f}_{b}=\mathbf{f}_{b}^{D}+\mathbf{f}_{b}^{I}=\left\{f_{21}, m_{1}, f_{22}, m_{2}\right\}, \\
\mathbf{f}_{a}^{D}=\left\{f_{11}^{D}, f_{12}^{D}\right\}, \\
\mathbf{f}_{b}^{D}=\left\{f_{21}^{D}, m_{1}^{D}, f_{22}^{D}, m_{2}^{D}\right\}, \\
\mathbf{f}_{a}^{I}=\left\{f_{11}^{I}, f_{22}^{I}\right\}, \\
\mathbf{f}_{b^{\prime}}^{I}=\left\{f_{21}^{I}, m_{1}^{I}, f_{22}^{I}, m_{2}^{I}\right\},
\end{gathered}
$$

where $\mathbf{f}_{i}(i=a, b)$ are the generalized force vectors corresponding to $\delta \mathbf{u}_{a}$ and $\delta \mathbf{u}_{b}$, respectively, $\mathbf{f}_{i}^{D}$ and $\mathbf{f}_{i}^{I}(i=a, b)$ are element deformation nodal force vector and inertia 
nodal force vector corresponding to $\mathbf{f}_{i}$, respectively, $V$ is the volume of the undeformed beam element, and $\delta \varepsilon_{11}$ is the variation of $\varepsilon_{11}$ in (2.13) corresponding to $\delta \mathbf{u}_{a}$ and $\delta \mathbf{u}_{b} . \sigma_{11}$ is the engineering stress. For linear elastic material, $\sigma_{11}=E \varepsilon_{11}$, where $E$ is Young's modulus. $\rho$ is the density, $\delta \mathbf{r}$ is the variation of $\mathbf{r}$ in (2.3) (referred to the current inertia element coordinate system) corresponding to $\delta \mathbf{u}_{a}$ and $\delta \mathbf{u}_{b}$, and $\mathbf{a}$ is the absolute acceleration in (2.15).

If the element size is chosen to be sufficiently small, the values of the deformation parameters of the deformed element defined in the current element coordinate system may always be much smaller than unity. Thus the higher-order terms of deformation parameters in the element internal nodal forces may be neglected. However, in order to include the nonlinear coupling among the bending and stretching deformations, the terms up to the second order of deformation parameters and their spatial derivatives are retained in element deformation nodal forces by consistent second-order linearization of $\delta \varepsilon_{11} \sigma_{11}$ in (2.22). Here, only infinitesimal-free vibration is considered, thus only the terms up to the first order of time derivatives of deformation parameters and their spatial derivatives are retained in element inertia nodal forces by consistent first-order linearization of $\delta \mathbf{r}^{t} \mathbf{a}$ in (2.22).

From (2.6) and (2.11), the variation of $\varepsilon_{11}$ in (2.13) may be expressed as

$$
\begin{gathered}
\delta \varepsilon_{11}=\delta \varepsilon_{c}-y \delta v_{, x x}, \\
\delta \varepsilon_{c}=\frac{1}{L}\left(\delta \mathbf{u}_{a}^{t} \mathbf{G}_{a}+\delta \mathbf{u}_{b}^{t} \mathbf{G}_{b}\right), \\
\delta v_{, x x}=\delta \mathbf{u}_{b}^{t} \mathbf{N}_{b}^{\prime \prime} .
\end{gathered}
$$

From (2.3), (2.6), and (2.12), $\delta \mathbf{r}$ the variation of $\mathbf{r}$ in (2.3) may be expressed as

$$
\begin{gathered}
\delta \mathbf{r}=\left\{\delta r_{1}, \delta r_{2}, \delta r_{3}\right\}=\left\{\delta x_{p}-y \delta v_{, x}, \delta v-y v_{, x} \delta v_{, x}, 0\right\} \\
\delta x_{p}=\delta \mathbf{u}_{a}^{t} \mathbf{N}_{a}+\frac{x}{L} \delta \mathbf{u}_{b}^{t} \mathbf{G}_{b}-\int_{0}^{x} v_{, x} \delta v_{, x} d x \\
\delta v_{, x}=\delta \mathbf{u}_{b}^{t} \mathbf{N}_{b}^{\prime} .
\end{gathered}
$$

Substituting (2.15)-(2.21) and (2.31)-(2.32) into (2.22), using $\int y d A=0$, neglecting the higher order terms, we may obtain

$$
\begin{aligned}
\mathbf{f}_{a}^{D}= & E A \varepsilon_{c} \mathbf{G}_{a}, \\
\mathbf{f}_{b}^{D}= & E I \int \mathbf{N}_{b}^{\prime \prime} v_{, x x} d x+f_{12}^{D} \int \mathbf{N}_{b}^{\prime} v_{, x} d x \\
\mathbf{f}_{a}^{I}= & \rho A \int \mathbf{N}_{a} \mathbf{N}_{a}^{t} d x \ddot{\mathbf{u}}_{a}+\Omega^{2} \rho A a_{o 1} \int \mathbf{N}_{a} d x-\Omega^{2} \rho A \int \mathbf{N}_{a}\left(\mathbf{N}_{a}^{t} \mathbf{u}_{a}+x\right) d x-2 \Omega \rho A \int \mathbf{N}_{a} \dot{v} d x, \\
\mathbf{f}_{b}^{I}= & \rho A \int \mathbf{N}_{b} \ddot{v} d x+\rho I \int \mathbf{N}_{b}^{\prime} \ddot{\ddot{v}, x} d x \\
& +\Omega^{2} \rho A a_{o 2} \int \mathbf{N}_{b} d x-\Omega^{2} \rho A \int \mathbf{N}_{b} v d x-\Omega^{2} \rho I \int \mathbf{N}_{b}^{\prime} v^{\prime} d x+2 \Omega \rho A \int \mathbf{N}_{b} \mathbf{N}_{a}^{t} d x \dot{\mathbf{u}}_{a} .
\end{aligned}
$$


where the range of integration for the integral $\int() d x$ in (2.34)-(2.36) is from 0 to $L, A$ is the cross section area, $I$ is moment of inertia of the cross section, $a_{o i}(i=1,2)$ are the $x_{i}$ components of $\mathbf{a}_{o}$ in (2.17). The underlined terms in (2.35) and (2.36) are the inertia nodal force corresponding to the steady-state deformation induced by the constant rotation.

\subsection{Element Matrices}

The element matrices considered are element tangent stiffness matrix, mass matrix, centripetal stiffness matrix, and gyroscopic matrix. The element matrices may be obtained by differentiating the element nodal force vectors in (2.33)-(2.36) with respect to nodal parameters and time derivatives of nodal parameters.

Using the direct stiffness method, the element tangent stiffness matrix may be assembled by the following submatrices:

$$
\begin{gathered}
\mathbf{k}_{a a}=\frac{\partial \mathbf{f}_{a}^{D}}{\partial \mathbf{u}_{a}}=\frac{E A}{L} \mathbf{G}_{a} \mathbf{G}_{a \prime}^{t} \\
\mathbf{k}_{a b}=\mathbf{k}_{b a}^{t}=\frac{\partial \mathbf{f}_{a}^{D}}{\partial \mathbf{u}_{b}}=\mathbf{0}, \\
\mathbf{k}_{b b}=\frac{\partial \mathbf{f}_{b}^{D}}{\partial \mathbf{u}_{b}}=E I \int \mathbf{N}_{b}^{\prime \prime} \mathbf{N}_{b}^{\prime \prime t} d x+f_{12}^{D} \int \mathbf{N}_{b}^{\prime} \mathbf{N}_{\mathbf{b}}^{\prime t} d x
\end{gathered}
$$

The element mass matrix may be assembled by the following submatrices:

$$
\begin{gathered}
\mathbf{m}_{a a}=\frac{\partial \mathbf{f}_{a}^{I}}{\partial \ddot{\mathbf{u}}_{a}}=\rho A \int \mathbf{N}_{a} \mathbf{N}_{a}^{t} d x \\
\mathbf{m}_{a b}=\mathbf{m}_{b a}^{t}=\frac{\partial \mathbf{f}_{a}^{I}}{\partial \ddot{\mathbf{u}}_{b}}=\mathbf{0}, \\
\mathbf{m}_{b b}=\frac{\partial \mathbf{f}_{b}^{I}}{\partial \ddot{\mathbf{u}}_{b}}=\rho A \int \mathbf{N}_{b} \mathbf{N}_{b}^{t} d x+\rho I\left(1-\varepsilon_{c}\right)^{2} \int \mathbf{N}_{b}^{\prime} \mathbf{N}_{b}^{\prime t} d x .
\end{gathered}
$$

The element centripetal stiffness matrix may be assembled by the following submatrices:

$$
\begin{gathered}
\mathbf{k}_{\Omega a a}=\frac{\partial \mathbf{f}_{a}^{I}}{\Omega^{2} \partial \mathbf{u}_{a}}=-\rho A \int \mathbf{N}_{a} \mathbf{N}_{a}^{t} d x, \\
\mathbf{k}_{\Omega a b}=\mathbf{k}_{\Omega a b}^{t}=\frac{\partial \mathbf{f}_{a}^{I}}{\Omega^{2} \partial \mathbf{u}_{b}}=\mathbf{0}, \\
\mathbf{k}_{\Omega b b}=\frac{\partial \mathbf{f}_{b}^{I}}{\Omega^{2} \partial \mathbf{u}_{b}}=-\rho A \int \mathbf{N}_{b} \mathbf{N}_{b}^{t} d x .
\end{gathered}
$$


Table 1: Dimensionless variables.

\begin{tabular}{|c|c|c|}
\hline & Variables & Dimensionless variables \\
\hline Coordinates & $x, X_{o}, Y_{o}$ & $\bar{x}=\frac{x}{L_{T}}, \bar{X}_{o}=\frac{X_{o}}{L_{T}}, \bar{Y}_{o}=Y_{o} / L_{T}$ \\
\hline Time & $t$ & $\tau=\frac{l}{L_{T}} \sqrt{\frac{L}{\rho}}$ \\
\hline $\begin{array}{l}\text { Length of beam } \\
\text { element }\end{array}$ & $L$ & $\bar{L}=L / L_{T}$ \\
\hline $\begin{array}{l}\text { Area moment of } \\
\text { inertia }\end{array}$ & $I$ & $\bar{I}=\frac{I}{A L_{T}^{2}}$ \\
\hline Radius of hub & $R$ & $\bar{R}=R / L_{T}$ \\
\hline Displacements & $u, v$ & $\bar{u}=u / L_{T}, \bar{v}=v / L_{T}$ \\
\hline $\begin{array}{l}\text { spatial derivatives } \\
\text { of displacement }\end{array}$ & $u^{\prime}, u^{\prime \prime}, v^{\prime}, v^{\prime \prime}$ & $\bar{u}^{\prime}=\frac{\partial \bar{u}}{\partial \bar{x}}=u^{\prime}, \bar{u}^{\prime \prime}=\frac{\partial^{2} \bar{u}}{\partial \bar{x}^{2}}=L_{T} u^{\prime \prime}, \bar{v}^{\prime}=\frac{\partial \bar{v}}{\partial \bar{x}}=v^{\prime}, \bar{v}^{\prime \prime}=\frac{\partial^{2} \bar{v}}{\partial \bar{x}^{2}}=L_{T} v^{\prime \prime}$ \\
\hline $\begin{array}{l}\text { Time derivatives of } \\
\text { displacement }\end{array}$ & $\dot{u}, \ddot{u}, \dot{v}, \ddot{v}$ & $\dot{\bar{u}}=\frac{\partial \bar{u}}{\partial \tau}=\dot{u} \sqrt{\frac{\rho}{E}}, \ddot{\bar{u}}=\frac{\partial^{2} \bar{u}}{\partial \tau^{2}}=L_{T} \frac{\rho}{E} \ddot{\bar{u}}, \dot{\bar{v}}=\frac{\partial \bar{v}}{\partial \tau}=\dot{v} \sqrt{\frac{\rho}{E}}, \ddot{\bar{v}}=\frac{\partial^{2} \bar{v}}{\partial \tau^{2}}=L_{T} \frac{\rho}{E} \ddot{v}$ \\
\hline Force and moment & $f_{i j}, m_{j}$ & $\bar{f}_{i j}=\frac{f_{i j}}{E A},(i=1,2 ; j=1,2)$ \\
\hline Angular velocity & $\Omega$ & $k=\Omega L_{T} \sqrt{\rho / E}$ \\
\hline Natural frequency & $\omega$ & $K=\omega L_{T} \sqrt{\rho / E}$ \\
\hline
\end{tabular}

The element gyroscopic matrix may be assembled by the following submatrices:

$$
\begin{gathered}
\mathbf{c}_{a a}=\frac{\partial \mathbf{f}_{a}^{I}}{\Omega \partial \dot{\mathbf{u}}_{a}}=\mathbf{0}, \\
\mathbf{c}_{a b}=-\mathbf{c}_{b a}^{t}=\frac{\partial \mathbf{f}_{a}^{I}}{\Omega \partial \dot{\mathbf{u}}_{b}}=-2 \rho A \int \mathbf{N}_{a} \mathbf{N}_{b}^{t} d x, \\
\mathbf{c}_{b b}=\frac{\partial \mathbf{f}_{b}^{I}}{\Omega \partial \dot{\mathbf{u}}_{b}}=\mathbf{0 .}
\end{gathered}
$$

\subsection{Equations of Motion}

For convenience, the dimensionless variables defined in Table 1 are used here.

The dimensionless nonlinear equations of motion for a rotating beam with constant angular velocity may be expressed by

$$
\begin{gathered}
\boldsymbol{\varphi}=\mathbf{F}^{D}(\widehat{\mathbf{Q}})+\mathbf{F}^{I}\left(k^{2}, \widehat{\mathbf{Q}}, \dot{\widehat{\mathbf{Q}}}, \ddot{\widehat{Q}}\right)=\mathbf{0} \\
\widehat{\mathbf{Q}}=\mathbf{Q}_{s}+\mathbf{Q}(\tau)
\end{gathered}
$$

where $k$ and $\tau$ are dimensionless time and dimensionless angular speed of rotating beam, respectively, defined in Table 1. $\boldsymbol{\varphi}, \mathbf{F}^{D}$, and $\mathbf{F}^{I}$ are the dimensionless unbalanced force 
vector, the dimensionless deformation nodal force vector, and the dimensionless inertia nodal force vector of the structural system, respectively. $\mathbf{F}^{I}$ and $\mathbf{F}^{D}$ are assembled from the dimensionless element nodal force vectors, which are calculated using (2.33)-(2.36) and the dimensionless variables defined in Table 1 first in the current element coordinates and then transformed from element coordinate system to global coordinate system before assemblage

using standard procedure. $\widehat{\mathbf{Q}}$ is the dimensionless nodal displacement vector of the rotating beam, $\dot{\hat{\mathbf{Q}}}=\partial \widehat{\mathbf{Q}} / \partial \tau$ and $\ddot{\overrightarrow{\mathbf{Q}}}=\partial^{2} \widehat{\mathbf{Q}} / \partial \tau^{2}$ are the dimensionless nodal velocity vector and the dimensionless nodal acceleration vector of the rotating beam, respectively, $\mathbf{Q}_{s}$ is the dimensionless steady-state nodal displacement vector induced by constant dimensionless rotation speed $k$, and $\mathbf{Q}(\tau)$ is the time-dependent dimensionless nodal displacements vector caused by the free vibration of the rotating beam. Here only infinitesimal vibration is considered.

\subsection{Governing Equations for Steady-state Deformation}

For the steady-state deformations, $\mathbf{Q}(\tau)=\mathbf{0}$. Thus (2.41) can be reduced to nonlinear dimensionless steady-state equilibrium equations and expressed by

$$
\boldsymbol{\varphi}=\mathbf{F}_{s}^{D}\left(\mathbf{Q}_{s}\right)+k^{2} \mathbf{F}_{s}^{I}\left(\mathbf{Q}_{s}\right)=\mathbf{0},
$$

where $\mathbf{F}_{s}^{D}\left(\mathbf{Q}_{s}\right)$ and $k^{2} \mathbf{F}_{s}^{I}\left(\mathbf{Q}_{s}\right)$ are the dimensionless deformation nodal force vector and the dimensionless inertia nodal force (the centrifugal force) vector of the structural system corresponding to the dimensionless steady-state nodal displacement vector $\mathbf{Q}_{s}$, respectively. $k^{2} \mathbf{F}_{s}^{I}\left(\mathbf{Q}_{s}\right)$ is corresponding to the underlined terms of (2.35) and (2.36). Note that $k^{2} \mathbf{F}_{S}^{I}\left(\mathbf{Q}_{s}\right)$ is deformation dependent. Thus $k^{2} \mathbf{F}_{s}^{I}\left(\mathbf{Q}_{s}\right)$ should be updated at each new configuration.

Here, an incremental-iterative method based on the Newton-Raphson method is employed for the solution of nonlinear dimensionless steady-state equilibrium equations at different dimensionless rotation speed $k$. In this paper, a weighted Euclidean norm of the unbalanced force is employed for the equilibrium iterations and is given by

$$
\frac{\|\varphi\|}{k^{2} \sqrt{N}\left\|\mathbf{F}_{s}^{I}\right\|} \leq e_{\mathrm{tol}}
$$

where $N$ is number of the equations of the system and $e_{\mathrm{tol}}$ is a prescribed value of error tolerance. Unless otherwise stated, the error tolerance $e_{\text {tol }}$ is set to $10^{-5}$ in this study.

\subsection{Governing Equations for Free Vibration Measured from the Position of Steady-State Deformation}

Substituting (2.42) into (2.41) and setting the first-order Taylor series expansion of the unbalanced force vector $\boldsymbol{\varphi}$ around $\mathbf{Q}_{s}$ to zero, one may obtain the dimensionless governing equations for linear free vibration of the rotating beam measured from the position of the steady-state deformation as follows.

$$
\mathbf{M} \mathbf{Q}+\mathbf{C Q}+\left(\mathbf{K}+k^{2} \mathbf{K}_{\Omega}\right) \mathbf{Q}=\mathbf{0}
$$


where $\mathbf{M}, \mathbf{C}, \mathbf{K}$, and $\mathbf{K}_{\Omega}$ are dimensionless mass matrix, gyroscopic matrix, tangent stiffness matrix, and centripetal stiffness matrix of the rotating beam, respectively. $\mathbf{M}, \mathbf{C}, \mathbf{K}$, and $\mathbf{K}_{\Omega}$ are assembled from the dimensionless element mass matrix, gyroscopic matrix, tangent stiffness matrix, and centripetal stiffness matrix, which are calculated using (2.37)-(2.40) and the dimensionless variables defined in Table 1 first in the current element coordinates and then transformed from element coordinate system to global coordinate system before assemblage using standard procedure.

We will seek a solution of (2.45) in the form

$$
\mathbf{Q}=\left(\mathbf{Q}_{R}+i \mathbf{Q}_{I}\right) e^{i K \tau}
$$

where $i=\sqrt{-1}, K$ and $\tau$ are dimensionless natural frequency of rotating beam and dimensionless time defined in Table 1 , and $\mathbf{Q}_{R}$ and $\mathbf{Q}_{I}$ are real part and imaginary part of the vibration mode.

Substituting (2.46) into (2.45), one may obtain a set of homogeneous equations expressed by

$$
\begin{gathered}
\mathbf{H Z}=\mathbf{0}, \\
\mathbf{H}=\mathbf{H}(K, k)=\left[\begin{array}{cc}
\mathbf{K}+k^{2} \mathbf{K}_{\Omega}-K^{2} \mathbf{M} & k K \mathbf{C}^{t} \\
k K \mathbf{C} & \mathbf{K}+k^{2} \mathbf{K}_{\Omega}-K^{2} \mathbf{M}
\end{array}\right], \\
\mathbf{Z}=\left\{\mathbf{Q}_{R}, \mathbf{Q}_{I}\right\},
\end{gathered}
$$

where $\mathbf{H}(K, k)$ denotes $\mathbf{H}$ being a function of $K$ and $k$. Note that $\mathbf{H}$ is a symmetric matrix.

Equation (2.47) is a quadratic eigenvalue problem. For a nontrivial $\mathbf{Z}$, the determinant of matrix $\mathbf{H}$ in (2.47) must be equal to zero. The values of $K$ which make the determinant vanishes are called eigenvalues of matrix $\mathbf{H}$. The bisection method is used here to find the eigenvalues. Note that when $k=0,(2.47)$ will degenerate to a generalized eigenvalue problem.

\section{Numerical Examples}

To verify the accuracy of the present method and to investigate the steady deformation and the natural frequencies of rotating-inclined beams with different inclination angle $\alpha$, dimensionless radius of the hub $\bar{R}$, and slenderness ratios $\eta=L_{T} \sqrt{A / I}$ at different dimensionless angular velocities $k$, several dimensionless numerical examples are studied here.

For simplicity, only the uniform beam with rectangular cross section is considered here. The maximum steady-state axial strain $\varepsilon_{\max }$ of rotating beam is the sum of the maximum steady-state membrane strain $\varepsilon_{c}^{\max }$ and bending strain $\varepsilon_{b}^{\max }$, which occur at the root of the rotating beam. In practice, rotating structures are designed to operate in the elastic range of the materials. Thus, it is considered that $\varepsilon_{\max } \leq \varepsilon_{y}$ (say 0.01) in this study. At the same dimensionless angular speed $k, \varepsilon_{\max }$ are different for rotating beams with different $\eta, \alpha$, and $\bar{R}$. Thus, the allowable $k$ are different for rotating beams with different $\eta, \alpha$, and $\bar{R}$ in this study. 
Table 2: Comparison of results for different cases $(\eta=20, \bar{R}=1.5)$.

\begin{tabular}{|c|c|c|c|c|c|c|c|c|c|c|c|c|}
\hline$\alpha$ & $k$ & & $\begin{array}{c}\varepsilon_{c}^{\max } \\
\left(10^{-3}\right)\end{array}$ & $\begin{array}{c}\varepsilon_{b}^{\max } \\
\left(10^{-3}\right)\end{array}$ & $\begin{array}{c}v_{\text {tip }} / L_{T} \\
\left(10^{-3}\right)\end{array}$ & $K_{1}$ & $K_{2}$ & $K_{3}(a)$ & $K_{4}$ & $K_{5}(a)$ & $K_{6}$ & $K_{7}(a)$ \\
\hline \multirow{10}{*}{$0^{\circ}$} & \multirow{5}{*}{0} & EA10 & 0 & 0 & 0 & .174788 & 1.05957 & 1.57241 & 2.82495 & 4.75610 & 5.19546 & 8.00214 \\
\hline & & EA50 & 0 & 0 & 0 & .174787 & 1.05953 & 1.57086 & 2.82431 & 4.71413 & 5.19120 & 7.86206 \\
\hline & & EA100 & 0 & 0 & 0 & .174787 & 1.05953 & 1.57081 & 2.82431 & 4.71283 & 5.19119 & 7.85600 \\
\hline & & [24] & 0 & 0 & 0 & .17479 & 1.05953 & 1.57080 & 2.82431 & 4.71239 & 5.19119 & - \\
\hline & & [34] & 0 & 0 & 0 & .17580 & 1.10172 & 1.57080 & 3.08486 & 4.71239 & 6.04510 & - \\
\hline & \multirow{5}{*}{0.06} & EA10 & 6.93309 & 0 & 0 & .198616 & 1.08756 & 1.57615 & 2.85333 & 4.75729 & 5.22384 & 8.02928 \\
\hline & & EA50 & 7.15492 & 0 & 0 & .198514 & $=1.08726$ & 1.57616 & 2.85243 & 4.71534 & 5.21931 & 7.86274 \\
\hline & & EA100 & 7.18210 & 0 & 0 & 198511 & 1.08726 & 1.57457 & 2.85242 & 4.71403 & 5.21930 & 7.85669 \\
\hline & & [24] & 7.20000 & 0 & 0 & .19862 & 1.08760 & 1.57455 & 2.85276 & 4.71360 & 5.21962 & - \\
\hline & & LAS & 7.20000 & 0 & 0 & - & - & - & - & - & - & - \\
\hline \multirow{4}{*}{$5^{\circ}$} & \multirow{4}{*}{0.03} & EA10 & 1.72680 & 1.93098 & 5.47630 & .1810 & 51 & 1.57 & 2.83 & 4.75 & 5.20256 & 8.00889 \\
\hline & & EA50 & 1.78195 & 1.93546 & 5.47699 & 181021 & 1.06651 & 1.57180 & 2.83136 & 4.71443 & 5.19823 & 7.86221 \\
\hline & & EA100 & 1.78870 & 1.93560 & 5.47701 & .181020 & 1.06651 & 1.57175 & 2.83136 & 4.71312 & 5.19822 & 7.85616 \\
\hline & & LAS & 1.79486 & 2.03794 & 5.88301 & - & - & - & - & - & - & - \\
\hline \multirow{4}{*}{$30^{\circ}$} & \multirow{4}{*}{0.01} & EA10 & .173298 & 1.29008 & 3.72294 & .175410 & 1.06028 & 1.57252 & 2.82567 & 4.75613 & 5.19619 & 8.00281 \\
\hline & & EA50 & .178615 & 1.29224 & 3.72299 & .175407 & 1.06024 & $=1.57097$ & 2.82503 & 4.71416 & 5.19191 & 7.86207 \\
\hline & & EA100 & .179264 & 1.29231 & 3.72300 & .175407 & 1.06024 & 1.57092 & 2.82503 & 4.71285 & 5.19190 & 7.85601 \\
\hline & & LAS & .179904 & 1.29904 & 3.75000 & - & - & - & - & - & - & - \\
\hline \multirow{4}{*}{$90^{\circ}$} & \multirow{4}{*}{0.01} & EA10 & .0500345 & 2.59364 & 7.49504 & .174836 & 1.05978 & 1.57253 & 2.82520 & 4.75612 & 5.19573 & 8.00229 \\
\hline & & EA50 & .0500384 & 2.59784 & 7.49506 & .174835 & 1.05974 & $=1.57098$ & 2.82456 & 4.71415 & 5.19145 & 7.86205 \\
\hline & & EA100 & .0500216 & 2.59797 & 7.49507 & .174835 & 1.05974 & 1.57093 & 2.82456 & 4.71284 & 5.19144 & 7.85599 \\
\hline & & LAS & .0500000 & 2.59807 & 7.50000 & - & - & - & - & - & - & - \\
\hline
\end{tabular}

To investigate the effect of the lateral deflection on the steady-state deformation and the natural frequency of rotating Euler beams, here cases with and without considering the lateral deflection are considered. The corresponding elements are referred to as EA element and EB element, respectively. For EA element, all terms in (2.33)-(2.40) are considered; for EB element, all terms in (2.33)-(2.40) are considered except the underlined terms in (2.36), which are the lateral inertia nodal force corresponding to the steady-state deformation induced by the constant rotation. In this section, $v_{\text {tip }} / L_{T}$ denotes the dimensionless lateral tip deflection of the steady-state deformation; $K_{i}$ denotes the $i$ th dimensionless natural frequency of the rotating beam and denote that the corresponding vibration mode is lateral vibration at $k=0$; in all tables, the entries with " $(a)$ " denotes that the corresponding vibration mode is axial vibration at $k=0$.

The example first considered is the rotating-inclined beams with dimensionless radius of the hub $\bar{R}=1.5$, inclination angle $\alpha=0^{\circ}, 5^{\circ}, 30^{\circ}, 90^{\circ}$, and slenderness ratios $\eta=20,1000$. The present results are shown in Tables 2 and 3 together with some results available in the literature. In Tables 2 and 3, EAn, $n=10,50,100$, denote that $n$ equal EA elements are used for discretization, and LAS denotes the linear analytical solution of the steady-state deformation. It can be seen that for higher natural frequencies of lateral vibration, the discrepancy between the present results and the analytical solutions given in [34], in which the rotary inertia is not considered, increases with decrease of the slenderness ratio. It seems that the effect of 
Table 3: Comparison of results for different cases $(\eta=1000, \bar{R}=1.5)$.

\begin{tabular}{|c|c|c|c|c|c|c|c|c|c|c|c|c|}
\hline$\alpha$ & $k$ & & $\begin{array}{c}\varepsilon_{c}^{\max } \\
\left(10^{-3}\right)\end{array}$ & $\begin{array}{c}\varepsilon_{b}^{\max } \\
\left(10^{-3}\right)\end{array}$ & $v_{\text {tip }} / L_{T}$ & $\begin{array}{c}K_{1} \\
\left(10^{-2}\right)\end{array}$ & $\begin{array}{c}K_{2} \\
\left(10^{-1}\right)\end{array}$ & $\begin{array}{c}K_{3} \\
\left(10^{-1}\right)\end{array}$ & $K_{4}$ & $K_{5}$ & $K_{6}$ & $K_{7}$ \\
\hline \multirow{10}{*}{$0^{\circ}$} & \multirow{5}{*}{0} & EA10 & 0 & 0 & 0 & .351601 & .220349 & .617105 & .121008 & 200340 & .300117 & .421052 \\
\hline & & EA50 & 0 & 0 & 0 & .351601 & .220341 & .616949 & .120893 & 199838 & .298509 & .416903 \\
\hline & & EA100 & 0 & 0 & 0 & .351601 & .220341 & .616948 & .120893 & .199837 & .298506 & .416896 \\
\hline & & [24] & 0 & 0 & 0 & .352 & .2203 & .6169 & .12089 & .19984 & .29851 & - \\
\hline & & [34] & 0 & 0 & 0 & .3516 & .22034 & .616972 & 120902 & - & - & - \\
\hline & \multirow{5}{*}{0.06} & EA10 & 6.93309 & 0 & 0 & 9.00457 & 2.50186 & 4.13423 & .591446 & .784725 & .992927 & 1.21760 \\
\hline & & EA50 & 7.15492 & 0 & 0 & 8.96239 & 2.47424 & 4.06068 & .580524 & .771309 & .976120 & 1.19365 \\
\hline & & EA100 & 7.18210 & 0 & 0 & 8.96152 & 2.47312 & 4.05756 & .580088 & .770833 & .975634 & 1.19316 \\
\hline & & [24] & 7.20000 & 0 & 0 & 8.952 & 2.4708 & 4.0536 & .57955 & .77017 & .97486 & - \\
\hline & & LAS & 7.20000 & 0 & 0 & - & - & - & - & - & - & - \\
\hline \multirow{4}{*}{$5^{\circ}$} & \multirow{4}{*}{0.03} & EA10 & 1.73113 & 3.88303 & 71 & 4.54 & 1.27 & 2.17 & 098 & .443024 & .577262 & .726698 \\
\hline & & EA50 & 1.78396 & 6.00526 & .0838194 & 4.53348 & 1.26220 & 2.15028 & .319777 & .439167 & .572464 & .719965 \\
\hline & & EA100 & 1.78936 & 6.20203 & .0838218 & 4.53320 & 1.26179 & 2.14942 & .319678 & .439068 & .572368 & .719873 \\
\hline & & LAS & 1.79486 & 101.897 & 14.70753 & - & - & - & - & - & - & - \\
\hline \multirow{4}{*}{$30^{\circ}$} & \multirow{4}{*}{0.008} & EA10 & .117174 & 8.73588 & .429688 & 1.29068 & .405580 & .836390 & .143462 & .221484 & .319586 & .439110 \\
\hline & & EA50 & .114341 & 9.36150 & .429979 & 1.28848 & .404156 & .836065 & .143631 & .221433 & .318254 & .434653 \\
\hline & & EA100 & .113410 & 9.38784 & .429986 & 1.28840 & .404108 & .836056 & .143643 & .221458 & .318289 & .434691 \\
\hline & & LAS & .115138 & 41.5692 & 6.00000 & - & - & - & - & - & - & - \\
\hline \multirow{4}{*}{$90^{\circ}$} & \multirow{4}{*}{0.003} & EA10 & 00632587 & 8.11012 & .747138 & .561367 & .232168 & .566051 & .113317 & .190889 & .289637 & .409722 \\
\hline & & EA50 & 00388224 & 8.15298 & .747250 & .560585 & .232182 & .566299 & .113204 & .190326 & .287895 & .405359 \\
\hline & & EA100 & .00351740 & 8.15396 & .747254 & .560558 & .232181 & .566306 & .113202 & .190322 & .287886 & .405342 \\
\hline & & LAS & .00450000 & 11.6913 & 1.68750 & - & - & - & - & - & - & - \\
\hline
\end{tabular}

the rotary inertia on the higher natural frequencies of the Euler beam is not negligible when the slenderness ratio is small. It can be seen from Tables 2 and 3 that the differences between the results of EA50 and EA 100 are negligible for all cases studied. Thus, in the rest of the section, all numerical results are obtained using 50 equal elements. For $\alpha=0$, and $k \neq 0$, the steady-state deformation is axial deformation only as expected. The analytical solution of the maximum steady-state membrane strain $\varepsilon_{c}^{\max }=k^{2}(\bar{R} \cos \alpha+1 / 2)$ given in [15] and the linear solution are identical. It can be seen that at the same dimensionless angular speed $k, \varepsilon_{c}^{\max }$ is independent of the slenderness ratio $\eta$. Thus, for $\alpha=0$, the allowable $k$ is limited by $\varepsilon_{c}^{\max }$ and is the same for the rotating beam with different slenderness ratio $\eta$. Very good agreement is observed between the natural frequencies obtained by the present study and those given in [24], which are obtained using the power series method. It can be seen from Table 3 that for slenderness ratio $\eta=1000$, with increase of the inclination angle $\alpha$, the values of $\varepsilon_{b}^{\max }$ and $v_{\text {tip }} / L_{T}$ increase significantly and the value of the allowable dimensionless angular speed $k$ decreases significantly. Comparing $\varepsilon_{b}^{\max }$ and $v_{\text {tip }} / L_{T}$ of EA with the results of linear analytical solution, respectively, it is found that the difference between the results of EA and LAS is insignificant for $\eta=20$ but is remarked for $\eta=1000$. These may be explained as follows. The centrifugal stiffening effect is significant for slender beam, and the lateral component of the centrifugal force in the rotating inclined beam decreases with the increase of the steady-state lateral deflection. 
Table 4: Dimensionless frequencies for rotating beam with different inclination angle $(\eta=70, \bar{R}=1, k=$ $5 / 70)$.

\begin{tabular}{lcccccccccc}
\hline$\alpha$ & \multicolumn{2}{c}{$\varepsilon_{c}^{\max }\left(10^{-3}\right)$} & $\varepsilon_{b}^{\max }$ & $v_{\text {tip }} / L_{T}$ & \multicolumn{3}{c}{$K_{1}$} & \multicolumn{3}{c}{$K_{2}$} \\
& $\mathrm{EA}$ & $\mathrm{EB}$ & $\mathrm{EA}$ & $\mathrm{EA}$ & $\mathrm{EA}$ & $\mathrm{EB}$ & {$[21]$} & $\mathrm{EA}$ & $\mathrm{EB}$ & {$[21]$} \\
\hline $0^{\circ}$ & 7.61582 & 7.61579 & 0 & 0 & .105565 & .105427 & .105 & .411754 & .410792 & .418 \\
$10^{\circ}$ & 7.53275 & 7.53893 & .021843 & .119537 & .105513 & .104869 & .105 & .411356 & .410001 & .417 \\
$20^{\circ}$ & 7.28556 & 7.31066 & .043509 & .236923 & .105359 & .103195 & .103 & .410160 & .407642 & .414 \\
$30^{\circ}$ & 6.88032 & 6.93792 & .064820 & .350059 & .105101 & .100399 & .100 & .408162 & .403758 & .410 \\
$40^{\circ}$ & 6.32700 & 6.43205 & .085602 & .456935 & .104737 & .0964721 & .096 & .405357 & .398421 & .405 \\
$50^{\circ}$ & 5.63927 & 5.80840 & .105681 & .555686 & .104264 & .0913941 & .091 & .401742 & .391733 & .398 \\
$60^{\circ}$ & 4.83425 & 5.08596 & .124890 & .644628 & .103679 & .0851262 & .085 & .397314 & .383830 & .390 \\
$70^{\circ}$ & 3.93214 & 4.28663 & .143064 & .722301 & .102976 & .0775919 & .077 & .392079 & .374876 & .381 \\
$80^{\circ}$ & 2.95575 & 3.43472 & .160043 & .787503 & .102151 & .0686418 & .068 & .386048 & .365073 & .371 \\
$90^{\circ}$ & 1.93010 & 2.55611 & .175673 & .839324 & .101193 & .0579597 & .057 & .379245 & .354659 & .361 \\
\hline
\end{tabular}

Table 5: Dimensionless frequencies for rotating beam with different inclination angle $(\eta=39, \bar{R}=1)$.

\begin{tabular}{|c|c|c|c|c|c|c|c|c|c|}
\hline $\begin{array}{ll}\alpha & k \\
\end{array}$ & $\varepsilon_{c}^{\max }\left(10^{-4}\right)$ & $\varepsilon_{b}^{\max }\left(10^{-3}\right)$ & $v_{\text {tip }} / L_{T}\left(10^{-3}\right)$ & $K_{1}\left(10^{-1}\right)$ & $K_{2}$ & $K_{3}$ & $K_{4}$ & $K_{5}$ & $K_{6}(a)$ \\
\hline 0 & 0 & 0 & 0 & .900168 & .559057 & 1.54325 & $1.57086(a)$ & 2.96396 & 4.71413 \\
\hline .010 & 1.48999 & 0 & 0 & .909817 & .560283 & 1.54452 & $1.57097(a)$ & 2.96528 & 4.71415 \\
\hline .020 & 5.96060 & 0 & 0 & .938126 & .563945 & 1.54830 & $1.57130(a)$ & 2.96926 & 4.71421 \\
\hline $0^{\circ} .030$ & 13.4138 & 0 & 0 & .983359 & .569996 & 1.55455 & $1.57190(a)$ & 2.97586 & 4.71432 \\
\hline .040 & 23.8528 & 0 & 0 & 1.04313 & .578359 & 1.56302 & $1.57296(a)$ & 2.98509 & 4.71449 \\
\hline .050 & 37.2822 & 0 & 0 & 1.11488 & .588935 & 1.57113 & $1.57704(a)$ & 2.99691 & 4.71472 \\
\hline .060 & 53.7078 & 0 & 0 & 1.19620 & .601604 & $1.57358(a)$ & 1.58936 & 3.01129 & 4.71502 \\
\hline .005 & .371545 & .073310 & .412011 & .902582 & .559363 & 1.54357 & $1.57089(a)$ & 2.96429 & 4.71414 \\
\hline \multirow{4}{*}{$5^{\circ}}$. & 1.48623 & 289977 & 1.62160 & .909786 & .560280 & 1.54449 & $1.57100(a)$ & 2.96528 & 4.71415 \\
\hline & 3.34417 & 640707 & 3.55360 & .921661 & .561805 & 1.54595 & $1.57126(a)$ & 2.96693 & 4.71416 \\
\hline & 5.94559 & 1.11150 & 6.09535 & .938019 & .563932 & 1.54784 & $1.57177(a)$ & 2.96925 & 4.71416 \\
\hline & 9.29075 & 1.68547 & 9.11211 & .958617 & .566654 & 1.55003 & 1.572 & 2.97222 & 4.71415 \\
\hline .030 & 13.3800 & 2.34473 & 12.4630 & .983170 & .569962 & 1.55233 & $1.57416(a)$ & 2.97585 & 4.71413 \\
\hline \multirow[b]{3}{*}{$30^{\circ} .006$} & .054292 & .067510 & .379956 & .900509 & .559102 & 1.54330 & $1.57087(a)$ & 2.96401 & 4.71413 \\
\hline & .217174 & .269614 & 1.51631 & .901534 & .559237 & 1.54341 & $1.57090(a)$ & 2.96415 & 4.71413 \\
\hline & .488657 & 605041 & 3.39858 & .903243 & .559461 & 1.54355 & $1.57103(a)$ & 2.96440 & 4.71412 \\
\hline \multirow{2}{*}{$\begin{array}{r}.008 \\
.010 \\
\end{array}$} & .868763 & 1.07170 & 6.00953 & .905635 & .559775 & 1.54362 & $1.57132(a)$ & 2.96474 & 4.71409 \\
\hline & 1.35752 & 1.66673 & 9.32547 & .908710 & .560177 & 1.54351 & $1.57191(a)$ & 2.96518 & 4.71403 \\
\hline \multirow{2}{*}{$\begin{array}{l}.002 \\
.004\end{array}$} & .019999 & .135091 & .760426 & .900208 & .559076 & 1.54327 & $1.57087(a)$ & 2.96398 & 4.71413 \\
\hline & .080009 & .540362 & 3.04080 & .900333 & .559132 & 1.54324 & $1.57098(a)$ & 2.9640 & 4.71412 \\
\hline $90^{\circ} .006$ & .180072 & 1.21579 & 6.83830 & .900554 & .559224 & 1.54295 & $1.57140(a)$ & 2.96415 & 4.71407 \\
\hline \multirow{2}{*}{$\begin{array}{l}.008 \\
.010\end{array}$} & .320253 & 2.16132 & 12.1478 & .900889 & .559349 & 1.54211 & $1.57246(a)$ & 2.96431 & 4.71394 \\
\hline & .500631 & 3.37678 & 18.9613 & .901363 & .559506 & 1.54041 & $1.57447(a)$ & 2.96451 & 4.71365 \\
\hline
\end{tabular}

To investigate the effect of the lateral deflection on the steady-state deformation and the natural frequency of rotating-inclined beams, the cases with and without considering the lateral deflection are studied for $\eta=70, \bar{R}=1$, and $k=5 / 70$. The present results are shown in Table 4 . The results transcribed from the figure given in [21], in which the steadystate lateral deflection and the rotary inertia are not considered, are also shown in Table 4 for 
Table 6: Dimensionless frequencies for rotating beam with different inclination angle $(\eta=50, \bar{R}=1)$.

\begin{tabular}{|c|c|c|c|c|c|c|c|c|c|c|}
\hline$\alpha$ & $k$ & $\varepsilon_{c}^{\max }\left(10^{-4}\right)$ & $\varepsilon_{b}^{\max }\left(10^{-3}\right)$ & $v_{\text {tip }} / L_{T}\left(10^{-3}\right)$ & $K_{1}\left(10^{-1}\right)$ & $K_{2}$ & $K_{3}$ & $K_{4}(a)$ & $K_{5}$ & $K_{6}$ \\
\hline \multirow{7}{*}{$0^{\circ}$} & 0 & 0 & 0 & 0 & .702550 & .437859 & 1.21530 & 1.57086 & 2.35176 & 3.82646 \\
\hline & .010 & 1.48998 & 0 & 0 & .714917 & .439441 & 1.21696 & 1.57096 & 2.35349 & 3.82822 \\
\hline & .020 & 5.96058 & 0 & 0 & .750712 & .444154 & 1.22192 & 1.57125 & 2.35868 & 3.83349 \\
\hline & .030 & 13.4137 & 0 & 0 & .806600 & .451898 & 1.23014 & 1.57173 & 2.36731 & 3.84227 \\
\hline & .040 & 23.8527 & 0 & 0 & .878442 & .462516 & 1.24155 & 1.57241 & 2.37932 & 3.85452 \\
\hline & .050 & 37.2820 & 0 & 0 & .962316 & .475813 & 1.25605 & 1.57328 & 2.39467 & 3.87021 \\
\hline & .060 & 53.7076 & 0 & 0 & 1.05500 & .491563 & 1.27352 & 1.57435 & 2.41329 & 3.88930 \\
\hline \multirow{6}{*}{$5^{\circ}$} & .005 & .371544 & .093761 & .674835 & .705653 & .438254 & 1.21571 & 1.57089 & 2.35219 & 3.82689 \\
\hline & .010 & 1.48623 & .368279 & 2.62905 & .714878 & .439437 & 1.21695 & 1.57096 & 2.35349 & 3.82821 \\
\hline & .015 & 3.34418 & .804892 & 5.66932 & .729982 & .441402 & 1.21900 & 1.57111 & 2.35565 & 3.83040 \\
\hline & .020 & 5.94560 & 1.37717 & 9.52433 & .750592 & .444136 & 1.22184 & 1.57134 & 2.35869 & 3.83347 \\
\hline & .025 & 9.29074 & 2.05594 & 13.8991 & .776240 & .447626 & 1.22547 & 1.57166 & 2.36258 & 3.83740 \\
\hline & .030 & 13.3799 & 2.81357 & 18.5209 & .806412 & .451854 & 1.22987 & 1.57208 & 2.36734 & 3.84221 \\
\hline \multirow{5}{*}{$30^{\circ}$} & .002 & .054292 & .086522 & .624206 & .702990 & .437917 & 1.21536 & 1.57087 & 2.35182 & 3.82652 \\
\hline & .004 & .217177 & .345191 & 2.48734 & .704308 & .438091 & 1.21554 & 1.57088 & 2.35202 & 3.82671 \\
\hline & .006 & .488671 & .773349 & 5.56121 & .706507 & .438381 & 1.21583 & 1.57093 & 2.35234 & 3.82704 \\
\hline & .008 & .868803 & 1.36665 & 9.79986 & .709585 & .438785 & 1.21621 & 1.57103 & 2.35280 & 3.82749 \\
\hline & .010 & 1.35760 & 2.11921 & 15.1409 & .713543 & .439301 & 1.21666 & 1.57120 & 2.35340 & 3.82806 \\
\hline \multirow{5}{*}{$90^{\circ}$} & .002 & .019999 & .173193 & 1.24980 & .702604 & .437883 & 1.21532 & 1.57087 & 2.35179 & 3.82648 \\
\hline & .004 & .080020 & .692767 & 4.99671 & .702772 & .437956 & 1.21539 & 1.57090 & 2.35188 & 3.82657 \\
\hline & .006 & .180129 & 1.55866 & 11.2327 & .703078 & .438073 & 1.21545 & 1.57103 & 2.35204 & 3.82670 \\
\hline & .008 & .320419 & 2.77065 & 19.9428 & .703561 & .438232 & 1.21544 & 1.57135 & 2.35228 & 3.82688 \\
\hline & .010 & .500997 & 4.32814 & 31.1020 & .704273 & .438426 & 1.21527 & 1.57200 & 2.35265 & 3.82709 \\
\hline
\end{tabular}

comparison. It can be seen from Table 4 that except $\alpha=0$, the values of $\varepsilon_{b}^{\max }$ are much larger than the yield strain for most engineering materials at $k=5 / 70$. Thus the results in Table 4 are only displayed for the purpose of comparisons between the results of EB and those given in [21]. There is a very good agreement between the natural frequencies obtained using the EB element and those given in [21]. Although the comparisons are beyond the yield point of most engineering materials, results of EA and EB show that the differences between the cases with and without considering the lateral deflection become apparent for the rotating-inclined beam with large inclination angle $\alpha$ at high-dimensionless angular speed. It can be seen from Table 4 that the difference between the natural frequencies of EA and EB is not significant for small $\alpha$, but the first natural frequency of EB is much smaller than that of EA for large $\alpha$. The natural frequencies of EA slightly decrease with increase of $\alpha$, but those of EB significantly decrease with increase of $\alpha$ for $\alpha \geq 50^{\circ}$. These may be partially attributed to the fact that the decrease of the centrifugal stiffening effect of the rotating-inclined beam caused by the increase of the inclination angle is alleviated by the increase of lateral deflection induced by the lateral centrifugal force.

To investigate the effect of angular speed on the steady-state deformation and natural frequency of rotating beams with different slenderness ratios and inclination angles, the following cases are considered: slenderness ratio $\eta=39,50,100,1000$, inclination angle $\alpha=0^{\circ}, 5^{\circ}, 30^{\circ}, 90^{\circ}$, and dimensionless radius of the rotating hub $\bar{R}=1$. Tables $5,6,7$, and 8 
Table 7: Dimensionless frequencies for rotating beam with different inclination angle $(\eta=100, \bar{R}=1)$.

\begin{tabular}{|c|c|c|c|c|c|c|c|c|c|c|}
\hline$\alpha$ & $k$ & $\varepsilon_{c}^{\max }\left(10^{-4}\right)$ & $\varepsilon_{b}^{\max }\left(10^{-3}\right)$ & $v_{\text {tip }} / L_{T}\left(10^{-3}\right)$ & $K_{1}\left(10^{-1}\right)$ & $K_{2}$ & $K_{3}$ & $K_{4}(a)$ & $K_{5}$ & $K_{6}$ \\
\hline \multirow{7}{*}{$0^{\circ}$} & 0 & .000000 & 0 & 0 & .351520 & .219989 & .614602 & 1.20047 & 1.57086 & 1.97619 \\
\hline & .010 & 1.48998 & 0 & 0 & .375696 & .223163 & 617963 & 1.20402 & 1.57096 & 1.97983 \\
\hline & .020 & 5.96058 & 0 & 0 & .439855 & .232418 & .627926 & 1.21458 & 1.57124 & 1.99072 \\
\hline & .030 & 13.4137 & 0 & 0 & .528670 & .247050 & .644140 & 1.23194 & 1.57172 & 2.00872 \\
\hline & .040 & 23.8527 & 0 & 0 & .630838 & .266138 & .666082 & 1.25578 & 1.57240 & 2.03361 \\
\hline & .050 & 37.2820 & 0 & 0 & .740095 & .288755 & .693127 & 1.28568 & 1.57327 & 2.06512 \\
\hline & .060 & 53.7076 & 0 & 0 & .853195 & .314093 & .724614 & 1.32116 & 1.57433 & 2.10288 \\
\hline \multirow{6}{*}{$5^{\circ}$} & .005 & .371547 & .184139 & 2.62891 & .357708 & .220785 & .615441 & 1.20136 & 1.57089 & 1.97710 \\
\hline & .010 & 1.48624 & .688585 & 9.52242 & .375636 & .223154 & 617940 & 1.20397 & 1.57105 & 1.97985 \\
\hline & .015 & 3.34416 & 1.40679 & 18.5127 & .403666 & .227045 & .622062 & 1.20826 & 1.57140 & 1.98446 \\
\hline & .020 & 5.94540 & 2.23838 & 27.6550 & .439745 & .232381 & .627773 & 1.21419 & 1.57195 & 1.99093 \\
\hline & .025 & 9.29010 & 3.11970 & 35.8627 & .481914 & .239066 & .635040 & 1.22176 & 1.57261 & 1.99922 \\
\hline & .030 & 13.3786 & 4.01960 & 42.7784 & .528564 & .246988 & .643816 & 1.23096 & 1.57329 & 2.00929 \\
\hline \multirow{5}{*}{$30^{\circ}$} & .002 & .054294 & .172596 & 2.48732 & .352403 & .220106 & .614725 & 1.20060 & 1.57087 & 1.97632 \\
\hline & .004 & .217197 & .683326 & 9.79956 & .355052 & .220456 & .615081 & 1.20096 & 1.57097 & 1.97675 \\
\hline & .006 & .488756 & 1.51188 & 21.5060 & .359467 & .221033 & .615636 & 1.20145 & 1.57136 & 1.97753 \\
\hline & .008 & .869003 & 2.62713 & 36.9471 & .365637 & .221832 & .616344 & 1.20197 & 1.57227 & 1.97873 \\
\hline & .010 & 1.35790 & 3.99065 & 55.3127 & .373528 & .222846 & .617163 & 1.20241 & 1.57395 & 1.98043 \\
\hline \multirow{5}{*}{$90^{\circ}$} & .002 & .020005 & .346383 & 4.99669 & .351634 & .220038 & .614651 & 1.20052 & 1.57089 & 1.97625 \\
\hline & .004 & .080103 & 1.38532 & 19.9425 & .352038 & .220178 & .614748 & 1.20053 & 1.57127 & 1.97654 \\
\hline & .006 & .180478 & 3.11497 & 44.6708 & .352915 & .220392 & .614736 & 1.20014 & 1.57288 & 1.97728 \\
\hline & .008 & .321226 & 5.52831 & 78.7975 & .354553 & .220651 & .614373 & 1.19878 & 1.57704 & 1.97891 \\
\hline & .010 & .502064 & 8.60794 & 121.586 & .357326 & .220924 & .613368 & 1.19581 & 1.58523 & 1.98201 \\
\hline
\end{tabular}

tabulate the maximum steady-state membrane strain and bending strain, dimensionless lateral tip deflection, and first six dimensionless natural frequencies for different $\eta$. It can be seen from Tables 5-8 that the values of $v_{\text {tip }} / L_{T}$ increase significantly with the increase of the dimensionless angular velocities $k$ and slenderness ratio $\eta$. However, the values of $v_{\text {tip }} / L_{T}$ are very small for $\eta=39$ and 50 . Because the stiffening effect of the centrifugal force is significant for slender beam, as expected, it can be seen from Table 8 that the lower natural frequencies of lateral vibration increase remarked with increase of the dimensionless angular speed for $\eta=1000$.

Figures 4-6 show the deformed configurations, axial displacements, and lateral displacements for the steady-state deformation of rotating beams with $\eta=100, \alpha=90^{\circ}$, and $\eta=1000, \alpha=5^{\circ}, 90^{\circ}$ at different dimensionless angular speeds. In Figures $4-6$, the $X_{1}$ and $X_{2}$ coordinates of the deformed configurations of rotating beam are present at the same scale, and $X_{1}^{0}$ denotes the global Lagrangian coordinate of the beam axis. Very large displacement and rotation are observed in Figure 6.

Figures 7-10 show the first six vibration modes for rotating beams with $=39, \alpha=0^{\circ}, 5^{\circ}$, and $\eta=1000, \alpha=5^{\circ}, 90^{\circ}$ at different dimensionless angular speeds. In Figures 7-10, $U$ and $V$ denote the $X_{1}$ and $X_{2}$ components of the vibration mode, respectively. The definitions of $U$ 
Table 8: Dimensionless frequencies for rotating beam with different inclination angle $(\eta=1000, \bar{R}=1)$.

\begin{tabular}{|c|c|c|c|c|c|c|c|c|c|c|}
\hline$\alpha$ & $k$ & $\varepsilon_{c}^{\max }\left(10^{-5}\right)$ & $\varepsilon_{b}^{\max }\left(10^{-3}\right)$ & $v_{\text {tip }} / L_{T}\left(10^{-2}\right)$ & $K_{1}\left(10^{-2}\right)$ & $K_{2}\left(10^{-1}\right)$ & $K_{3}\left(10^{-1}\right)$ & $K_{4}$ & $K_{5}$ & $K_{6}$ \\
\hline \multirow{7}{*}{$0^{\circ}$} & 0 & .000000 & 0 & 0 & .351601 & .220341 & .616949 & .120893 & .199838 & .298509 \\
\hline & .010 & 14.8998 & 0 & 0 & 1.32581 & .432338 & .886010 & 151855 & .233376 & .333736 \\
\hline & .020 & 59.6058 & 0 & 0 & 2.52842 & .766172 & 1.38410 & .216189 & .309541 & .419056 \\
\hline & .030 & 134.137 & 0 & 0 & 3.73648 & 1.11402 & 1.92511 & .289167 & .400408 & .525942 \\
\hline & .040 & 238.527 & 0 & 0 & 4.94495 & 1.46597 & 2.47903 & .364653 & .496007 & .640880 \\
\hline & .050 & 372.820 & 0 & 0 & 6.15267 & 1.81983 & 3.03912 & .441165 & .593428 & .759068 \\
\hline & .060 & 537.076 & 0 & 0 & 7.35907 & 2.17482 & 3.60301 & .518237 & .691707 & .878747 \\
\hline \multirow{6}{*}{$5^{\circ}$} & .005 & 3.71469 & .765147 & 5.97092 & .741097 & .289190 & .695401 & .129392 & .208701 & .307598 \\
\hline & .010 & 14.8600 & 1.67568 & 7.33938 & 1.32573 & .432307 & .885745 & .151766 & .233190 & .333432 \\
\hline & .015 & 33.4431 & 2.57966 & 7.79173 & 1.92549 & .595911 & 1.12500 & .182037 & .268266 & .371976 \\
\hline & .020 & 59.4713 & 3.46977 & 2050 & & .766160 & & .216153 & .309441 & .418839 \\
\hline & .025 & 92.9513 & 4.34189 & 8.15957 & 3.13220 & .939307 & 1.65211 & .252155 & .353965 & .470793 \\
\hline & .030 & 133.889 & 5.19316 & 8.25360 & 3.73641 & 1.11401 & 1.92507 & .289150 & .400356 & .525817 \\
\hline \multirow{4}{*}{$30^{\circ}$} & .002 & .542410 & 1.31449 & 16.2578 & .436039 & .231506 & .625674 & .121676 & .200527 & .299138 \\
\hline & .004 & 2.15709 & 3.45499 & 31.0603 & .628044 & .264068 & .655502 & .124160 & .202509 & .300711 \\
\hline & .006 & 4.84203 & 5.61881 & 37.4726 & .851755 & .312455 & .712896 & .129772 & .207598 & .305211 \\
\hline & .008 & 8.61268 & 7.78502 & 40.6536 & 1.08530 & .369468 & .788930 & .138176 & .215896 & .313013 \\
\hline \multirow{4}{*}{\multicolumn{2}{|c|}{$90^{\circ} .0020$}} & .012524 & .216407 & 3.11010 & .352489 & .220632 & .617142 & .120911 & .199852 & .298522 \\
\hline & & .050204 & .860794 & 12.1582 & .357434 & .221288 & .616084 & .120749 & .199640 & .298279 \\
\hline & & .112068 & 1.89528 & 25.7173 & .371925 & .221920 & .610342 & .119952 & .198654 & .297181 \\
\hline & & .194123 & 3.21653 & 40.7205 & .400095 & .222722 & .599365 & .118425 & .196788 & .295121 \\
\hline \multirow{2}{*}{\multicolumn{2}{|c|}{$\begin{array}{l}.0025 \\
.0030\end{array}$}} & .290775 & 4.68879 & 54.0272 & .440751 & .224723 & .587411 & .116669 & .194633 & .292734 \\
\hline & & .398355 & 6.21301 & 64.2905 & .489485 & .228876 & .578807 & .115177 & .192740 & .290595 \\
\hline & .0035 & .516056 & 7.74385 & 71.7152 & .542488 & .235452 & .575106 & .114128 & .191302 & .288900 \\
\hline
\end{tabular}

and $V$ are given by

$$
\begin{gathered}
U=\left(U_{R}^{2}+U_{I}^{2}\right)^{1 / 2} \operatorname{sign}\left(\sin \phi_{u}\right), \quad \sin \phi_{u}=\frac{U_{I}}{\left(U_{R}^{2}+U_{I}^{2}\right)^{1 / 2}}, \quad-\pi \leq \phi_{u} \leq \pi, \\
V=\left(V_{R}^{2}+V_{I}^{2}\right)^{1 / 2} \operatorname{sign}\left(\sin \phi_{v}\right), \quad \sin \phi_{v}=\frac{V_{I}}{\left(V_{R}^{2}+V_{I}^{2}\right)^{1 / 2}}, \quad-\pi \leq \phi_{v} \leq \pi, \\
\operatorname{sign}(x)= \begin{cases}1 & \text { for } x>0, \\
-1 & \text { for } x<0,\end{cases}
\end{gathered}
$$

where $U_{R}$ and $V_{R}$, and $U_{I}$ and $V_{I}$ are the $X_{1}$ and $X_{2}$ components of $\mathbf{Q}_{R}$ and $\mathbf{Q}_{I}$, real part and imaginary part of the vibration mode given in (2.47), respectively. $\phi_{u}$ and $\phi_{v}$ are phase angles. Mode $j(j=1-6)$ denotes the vibration mode dominated by the vibration mode corresponding to the $j$ th natural frequency of the nonrotating beam. It can be seen from Figures 7-10, and Tables 5 and 8 that all vibration modes shown in Figures 7-10 are lateral 


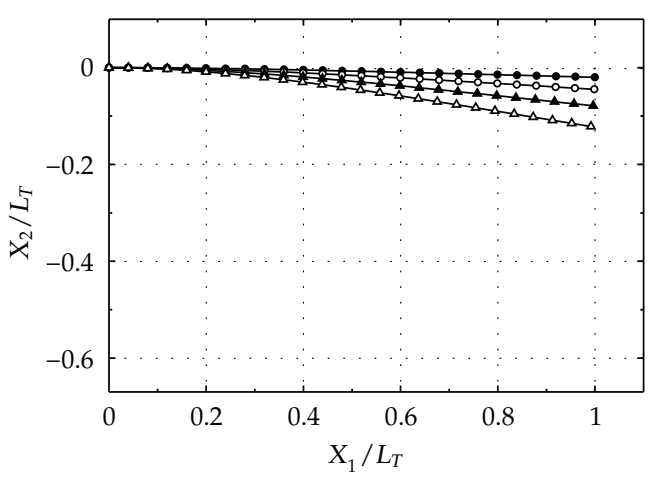

(a)

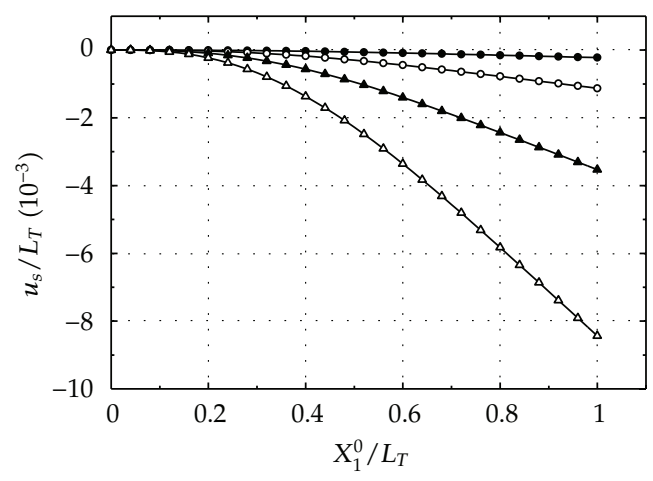

(b)

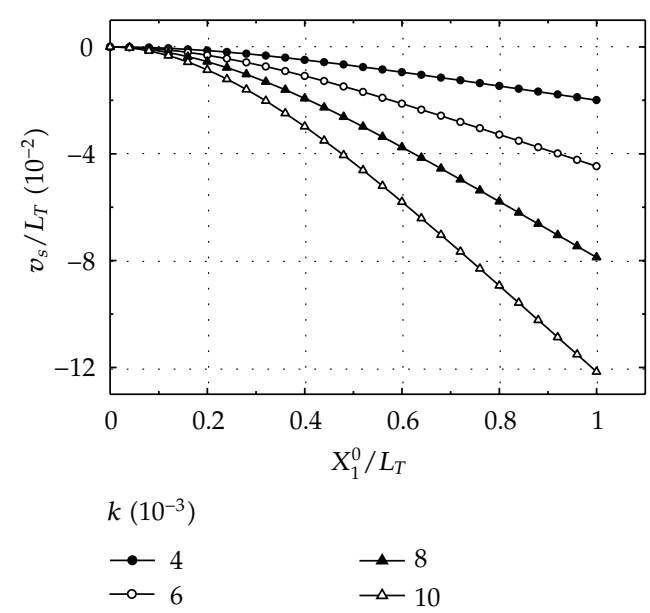

(c)

Figure 4: The steady-state deformation of rotating beam, (a) deformed configuration, (b) axial displacement, and (c) lateral displacement $\left(\eta=100, \bar{R}=1, \alpha=90^{\circ}\right)$.

vibration at $k=0$, except the fourth and the sixth vibration modes of $\eta=39$. Mode 3 is the third bending vibration mode, and mode 4 is the first axial vibration mode for $\eta=39$. Figure 11 shows the third and the fourth dimensionless natural frequencies for the rotating beam with $\eta=39$ at different dimensionless angular velocities. Because the third and the fourth natural frequencies are relatively close, frequency veering phenomenon [24] induced by the Coriolis force and the centrifugal force is observed in Figure 11. It can be seen from Figures 7 and 8 that the coupling of the axial and lateral vibration modes is very significant. Due to the effect of Coriolis force and the lateral steady-state deformation, the axial and lateral vibrations of rotating beam should be coupled. However, from the numerical results of this study, it is found that the coupling is negligible for rotating beam with small slenderness ratio if the corresponding natural frequencies of the axial and lateral vibrations are not close. Due to the steady-state lateral deformation, it can be seen from Figures 9 and 10 that when $k \neq 0$, all vibration modes consist of the $X_{1}$ and $X_{2}$ components. The difference between the vibration modes of rotating beam at different $k$ is very significant for $\eta=1000$. 


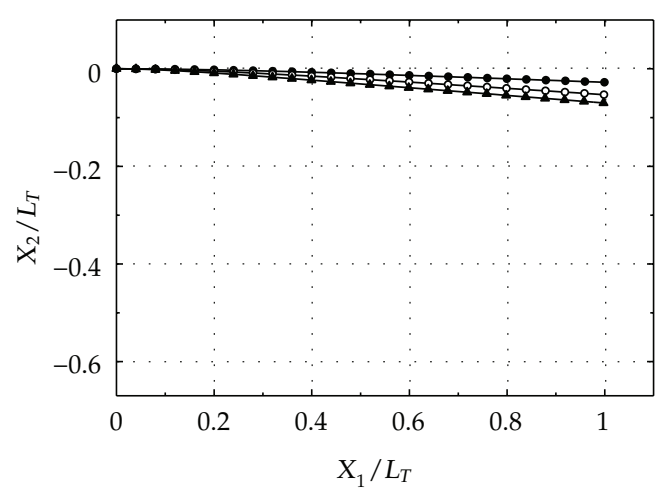

(a)

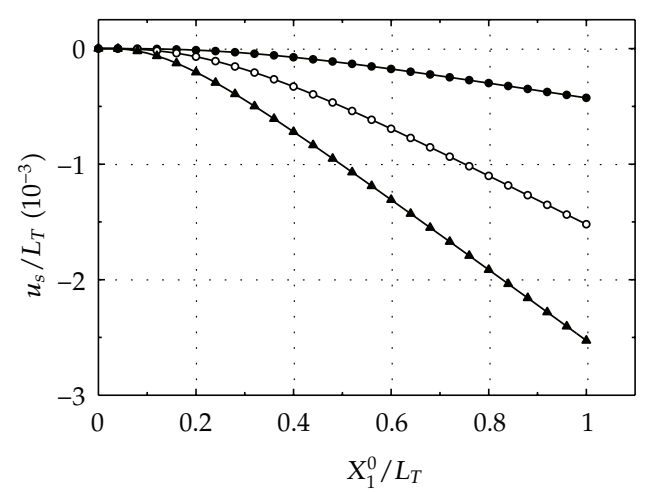

(b)

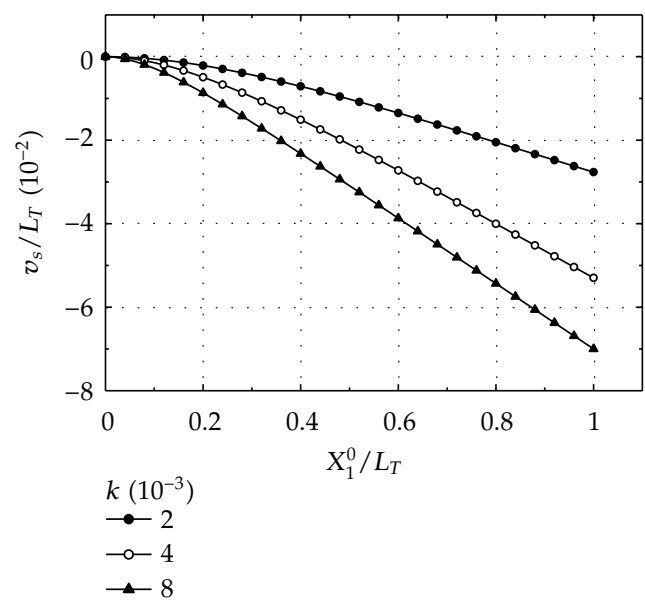

(c)

Figure 5: The steady-state deformation of rotating beam, (a) deformed configuration, (b) axial displacement, and (c) lateral displacement $\left(\eta=1000, \bar{R}=1, \alpha=5^{\circ}\right)$.

\section{Conclusions}

In this paper, a corotational finite element formulation combined with the rotating frame method and numerical procedure are proposed to derive the equations of motion for a rotating-inclined Euler beam at constant angular velocity. The element deformation and inertia nodal forces are systematically derived by the virtual work principle, the d'Alembert principle, and consistent second-order linearization of the fully geometrically nonlinear beam theory in the current element coordinates. The equations of motion of the system are defined in terms of an inertia global coordinate system which is coincident with a rotating global coordinate system rigidly tied to the rotating hub, while the total strains in the beam element are measured in an inertia element coordinate system which is coincident with a rotating element coordinate system constructed at the current configuration of the beam element. The rotating element coordinates rotate about the hub axis at the angular speed of the hub. The steady-state deformation and the natural frequency of infinitesimal-free vibration measured from the position of the corresponding steady-state deformation are 


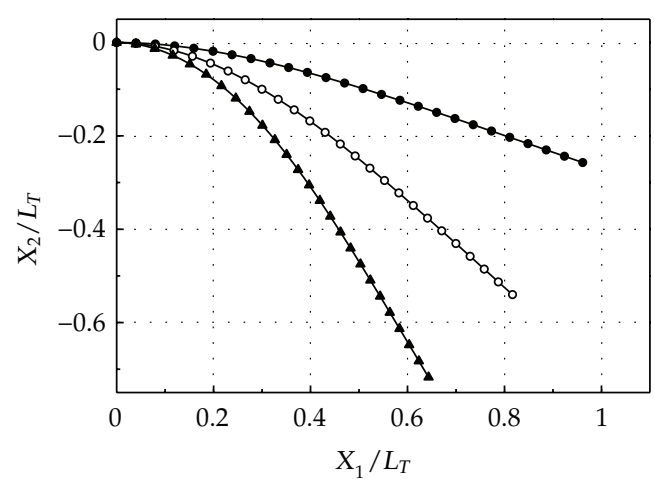

(a)

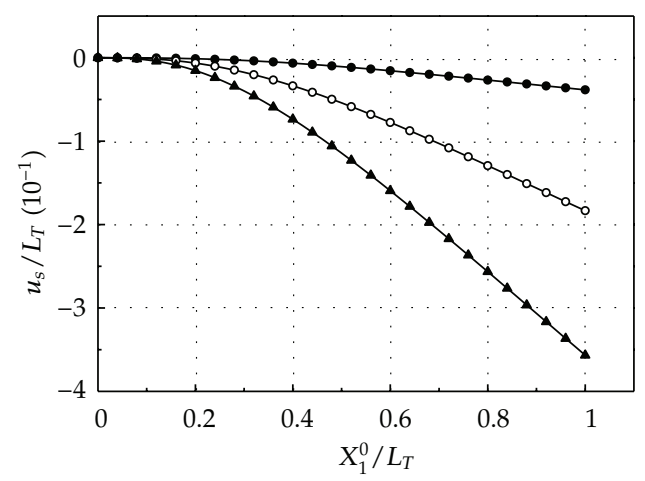

(b)

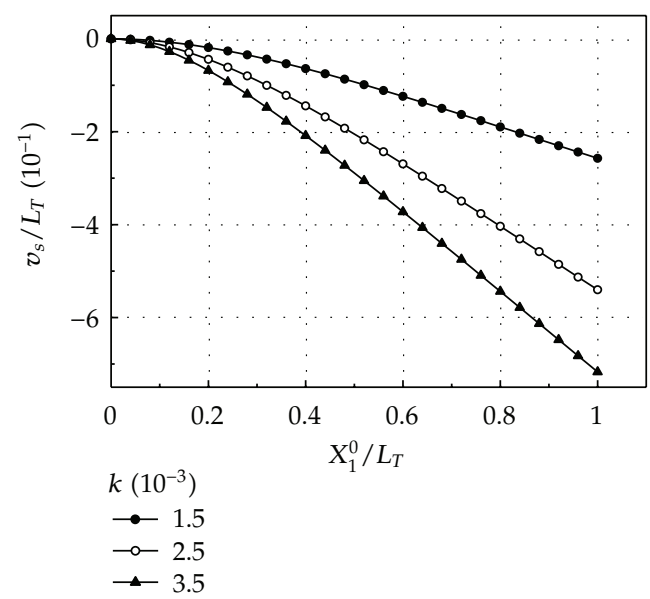

(c)

Figure 6: The steady-state deformation of rotating beam, (a) deformed configuration, (b) axial displacement, (c) lateral displacement $\left(\eta=1000, \bar{R}=1, \alpha=90^{\circ}\right)$.

investigated for rotating-inclined Euler beams with different inclination angles, slenderness ratios, and angular speeds of the hub.

The results of dimensionless numerical examples demonstrate the accuracy and efficiency of the proposed method. The present results show that the geometrical nonlinearities that arise due to steady-state lateral and axial deformations should be considered for the natural frequencies of the inclined-rotating beams. Due to the effect of the centrifugal stiffening, the lower dimensionless natural frequencies of lateral vibration increase remarked with increase of the dimensionless angular speed for slender beam. The decrease of the cen-trifugal stiffening effect of the rotating inclined beam caused by the increase of the inclination angle is alleviated by the increase of lateral deflection induced by the lateral centrifugal force. Due to effect of the Coriolis force and centrifugal stiffening, frequency veering phenomenon is observed when inclination angle $\alpha=0^{\circ}$, and two natural frequencies corresponding to axial vibration and lateral vibration are close.

Finally, it may be emphasized that, although the proposed method is only applied to the two dimensional rotating cantilever beams with inclination angle here, the present method can be easily extended to three dimensional rotating beams with precone and setting angle. 


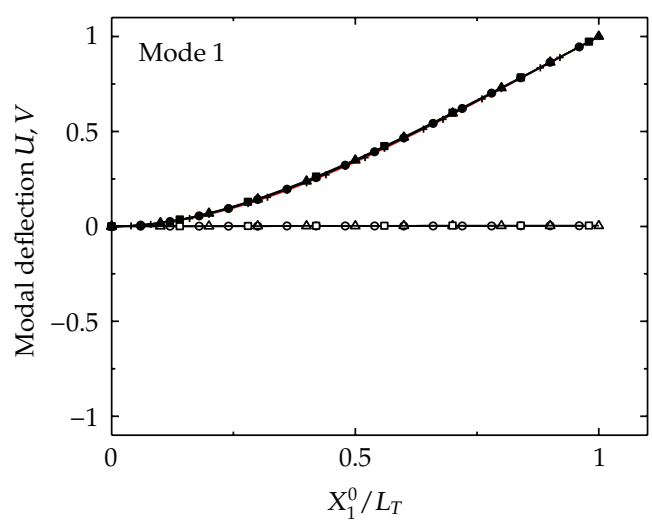

(a)

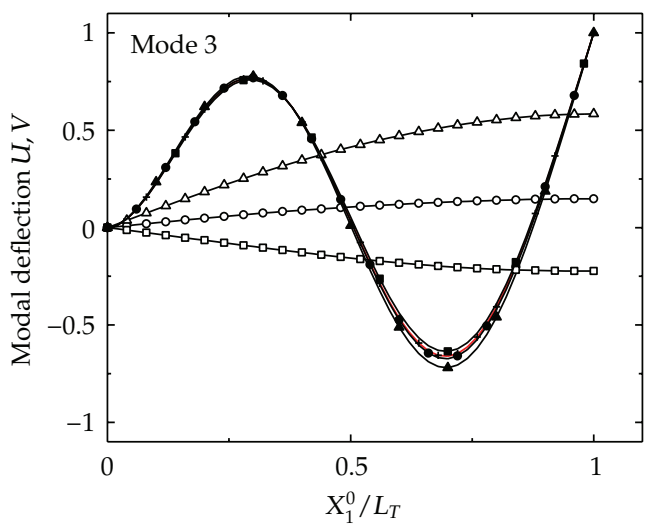

(c)

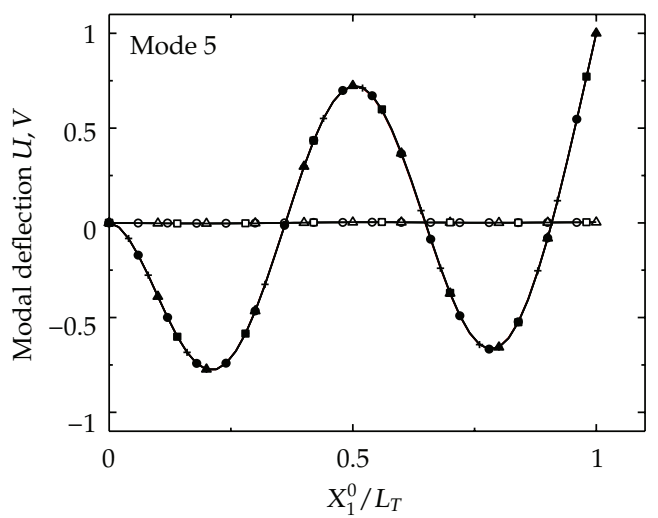

$$
\begin{array}{ll}
\stackrel{U}{\longrightarrow}(k=0) & \stackrel{V}{+}(k=0) \\
\multimap-(k=0.04) & \longrightarrow-(k=0.04) \\
\square-(k=0.05) & \longrightarrow(k=0.05) \\
\square-(k=0.06) & \longrightarrow-(k=0.06)
\end{array}
$$

(e)

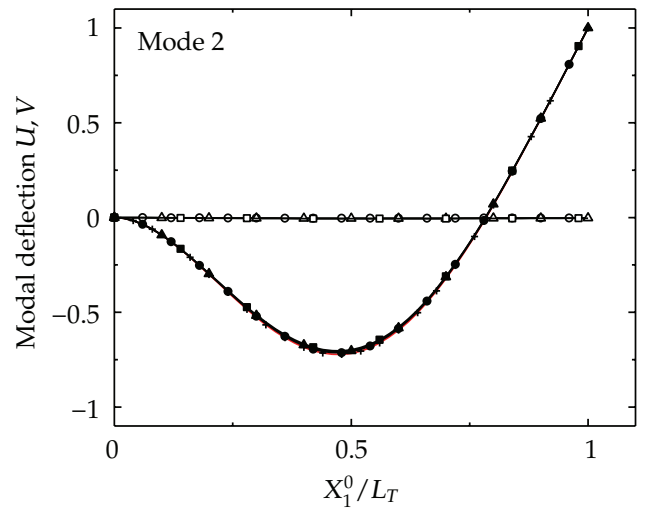

(b)

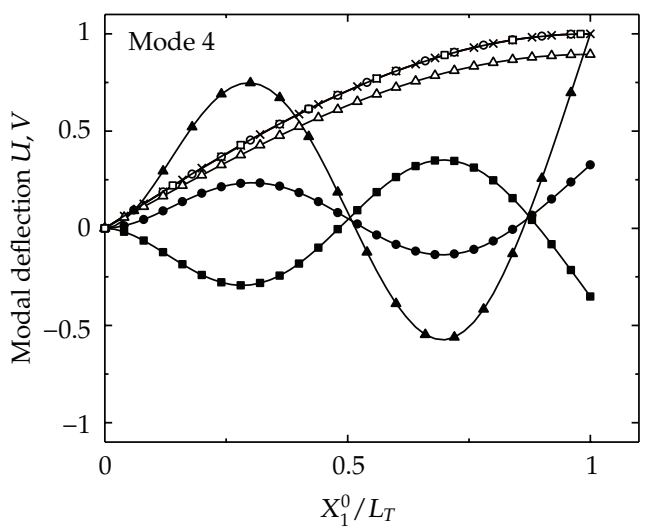

(d)

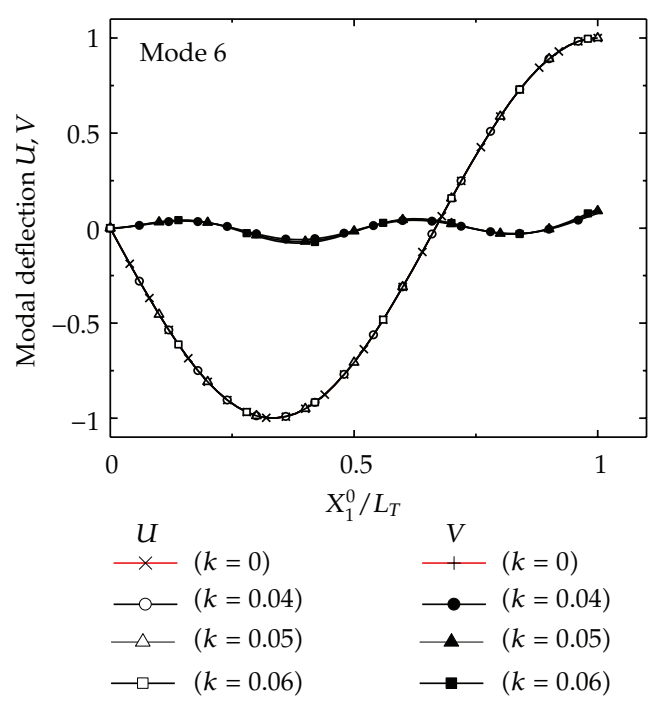

(f)

Figure 7: The first six vibration mode shapes of a rotating beam $\left(\eta=39, \bar{R}=1, \alpha=0^{\circ}\right)$. 


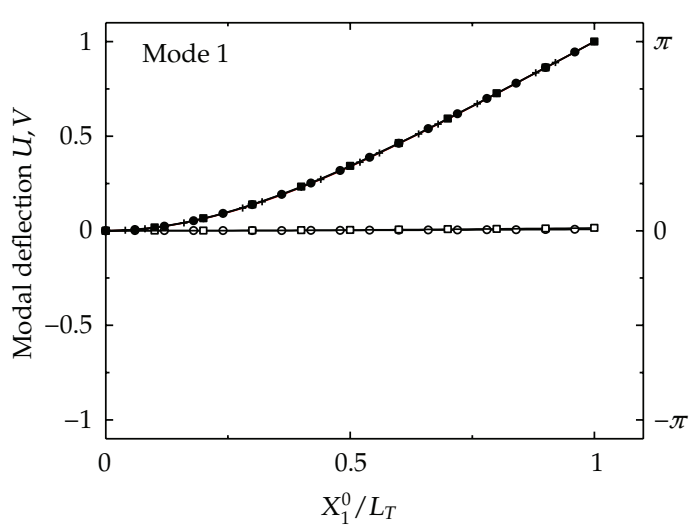

(a)

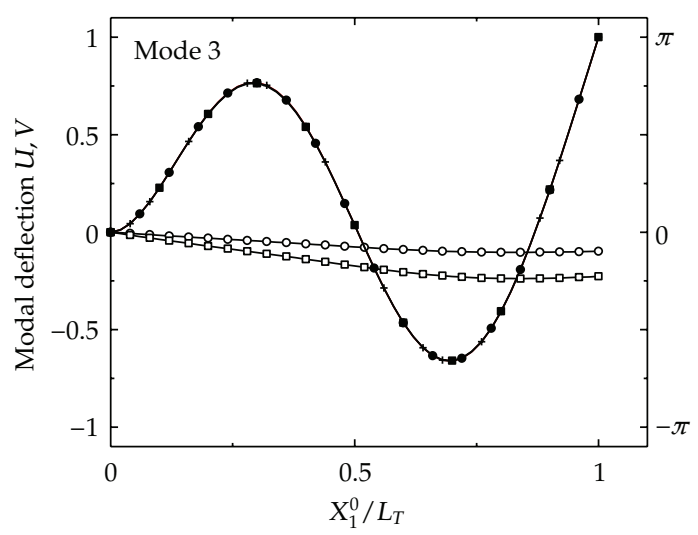

(c)

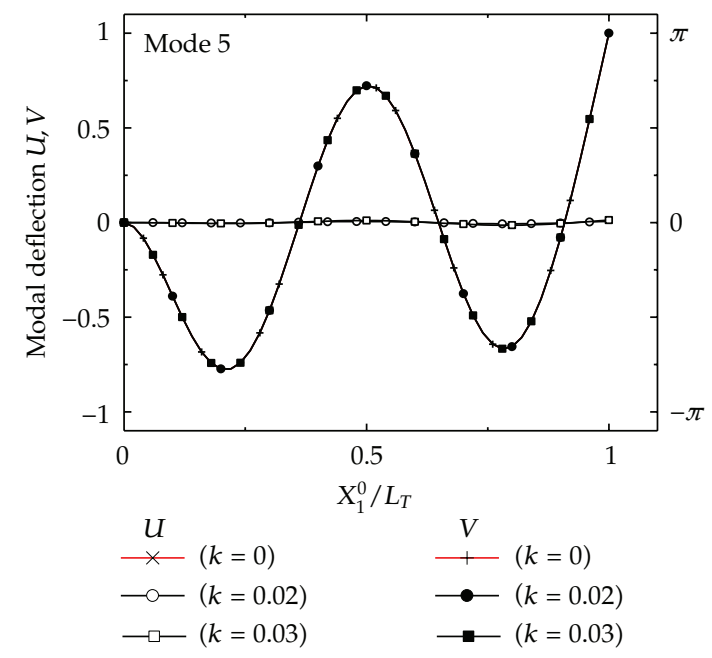

(e)

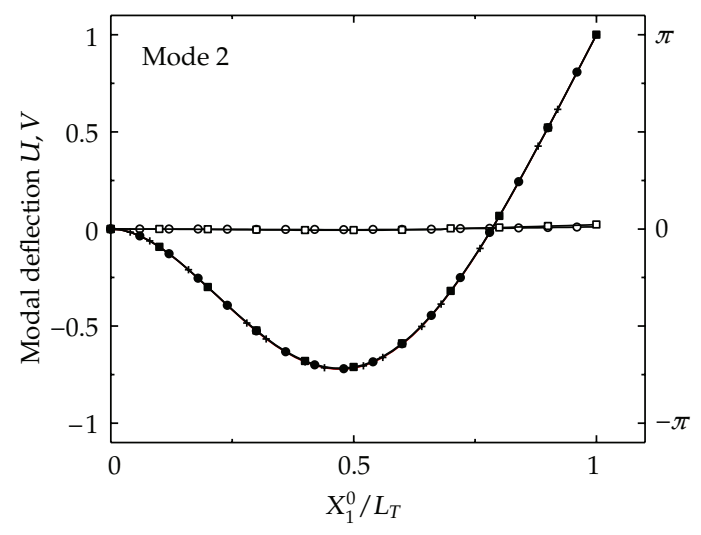

(b)

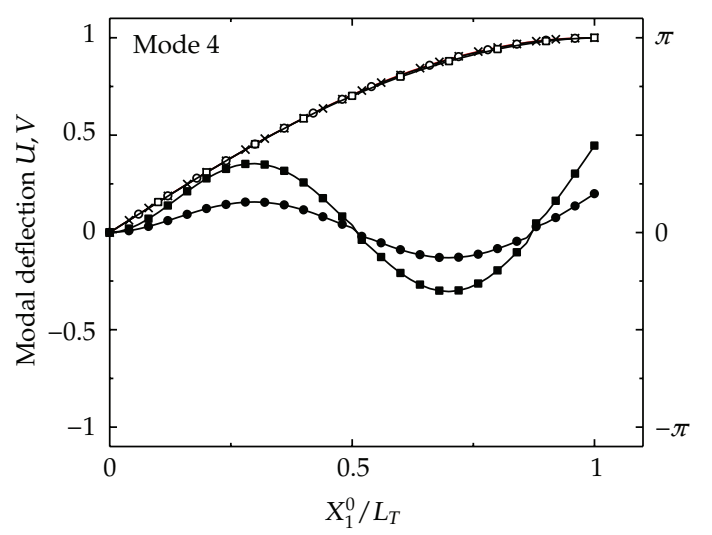

(d)

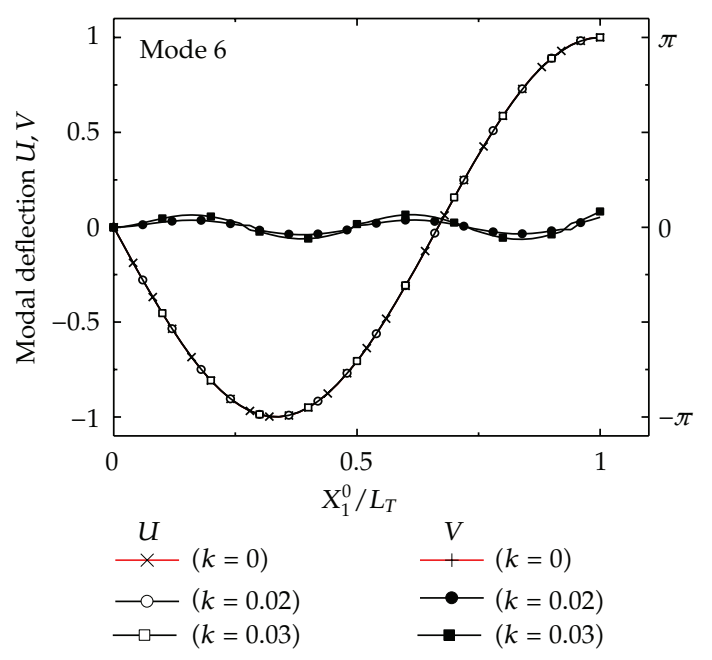

(f)

Figure 8: The first six vibration mode shapes of a rotating beam $\left(\eta=39, \bar{R}=1, \alpha=5^{\circ}\right)$. 


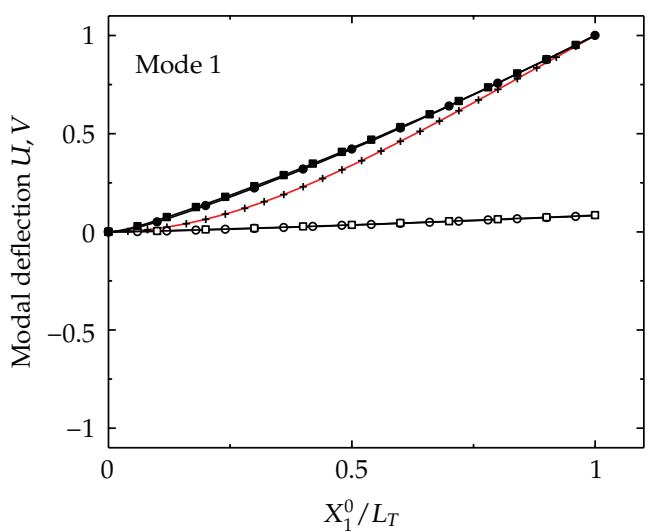

(a)

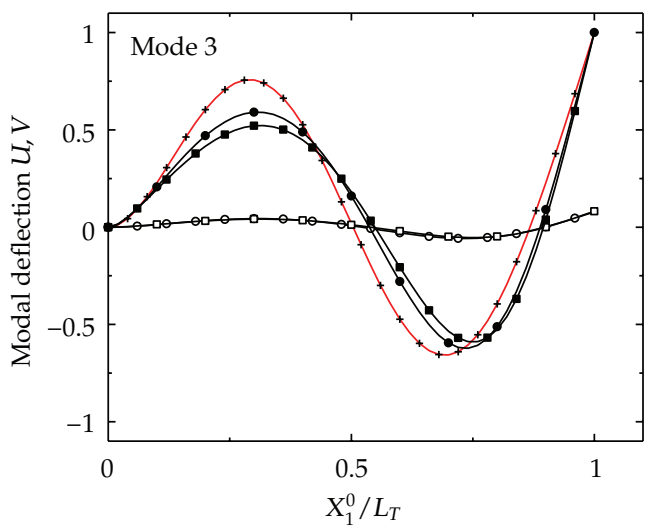

(c)

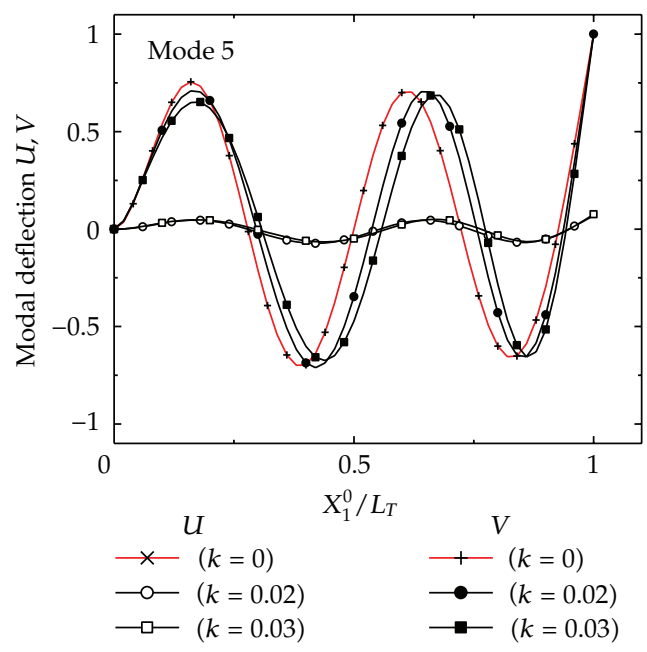

(e)

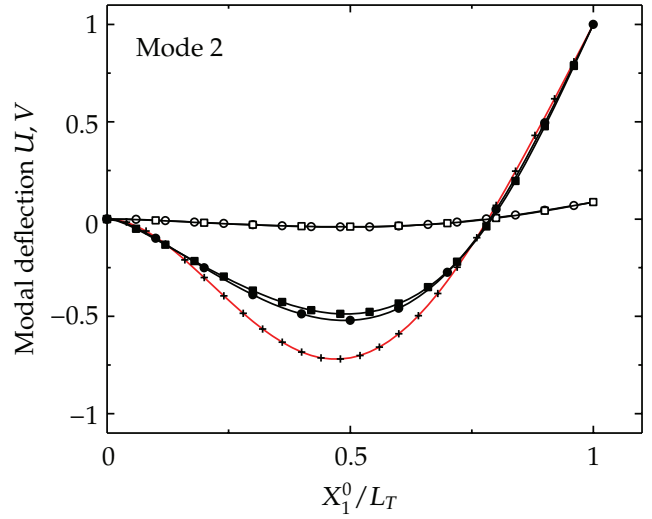

(b)

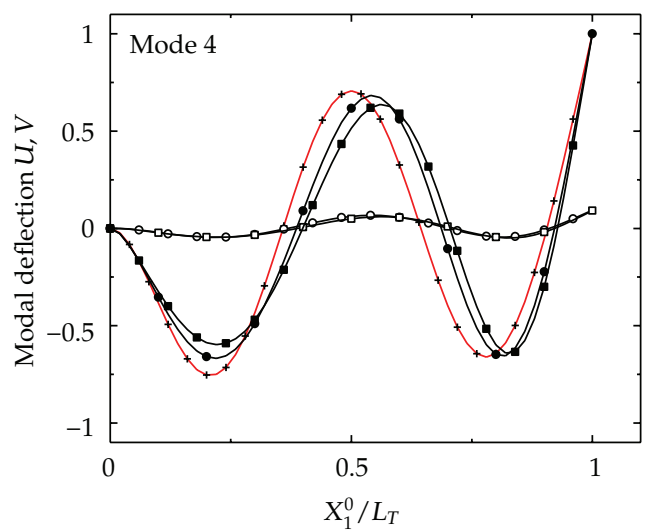

(d)

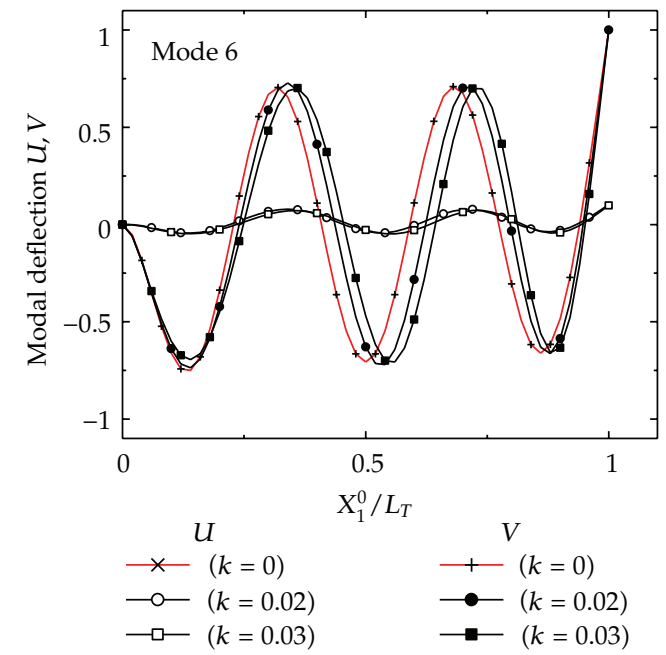

(f)

Figure 9: The first six vibration mode shapes of a rotating beam $\left(\eta=1000, \bar{R}=1, \alpha=5^{\circ}\right)$. 


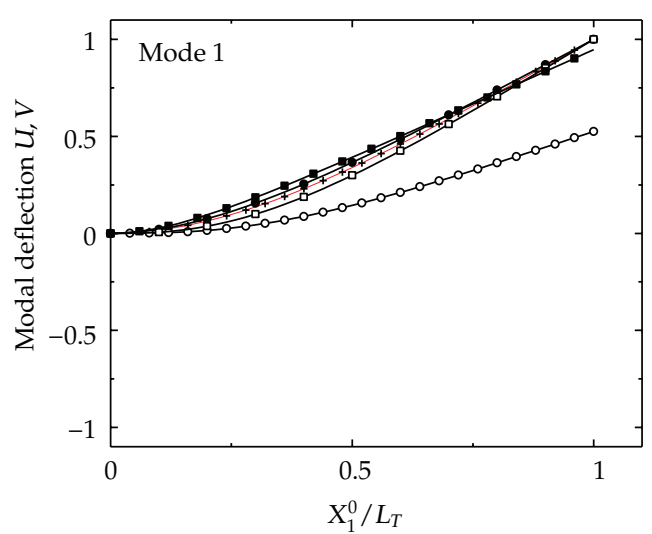

(a)

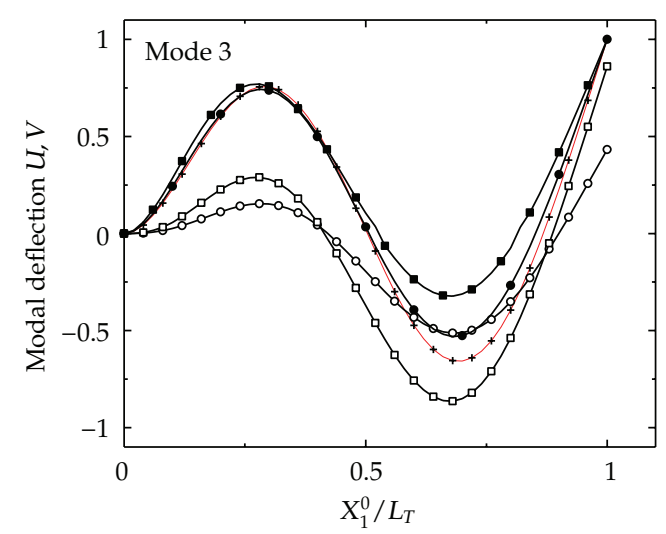

(c)

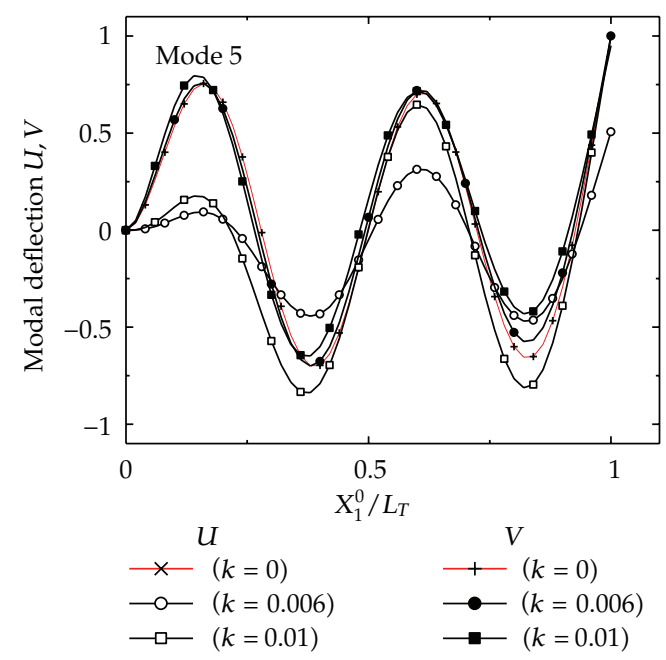

(e)

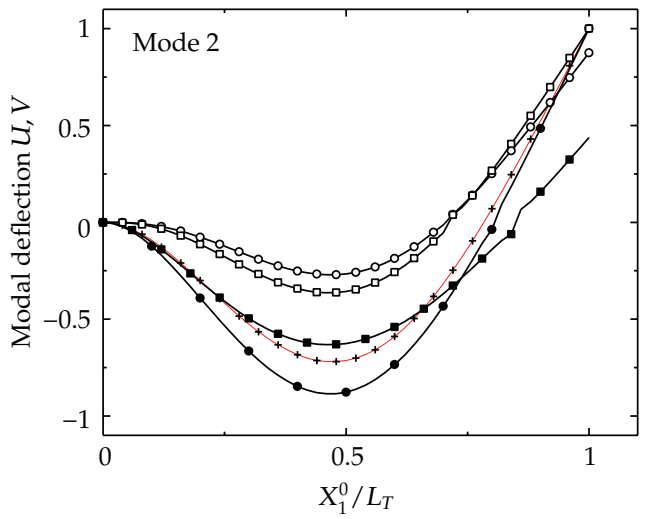

(b)

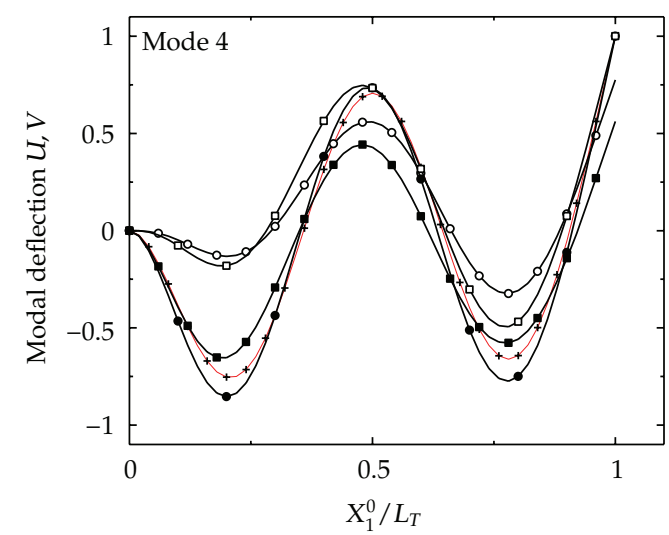

(d)

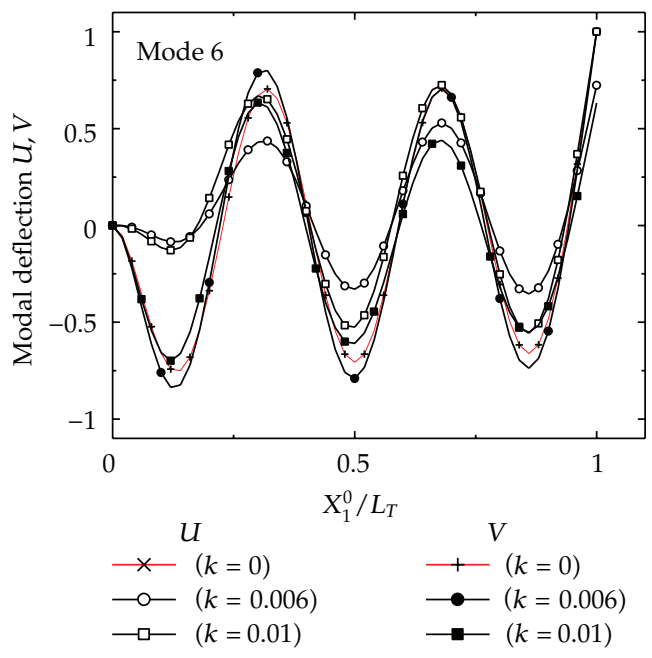

(f)

Figure 10: The first six vibration mode shapes of a rotating beam $\left(\eta=1000, \bar{R}=1, \alpha=90^{\circ}\right)$. 


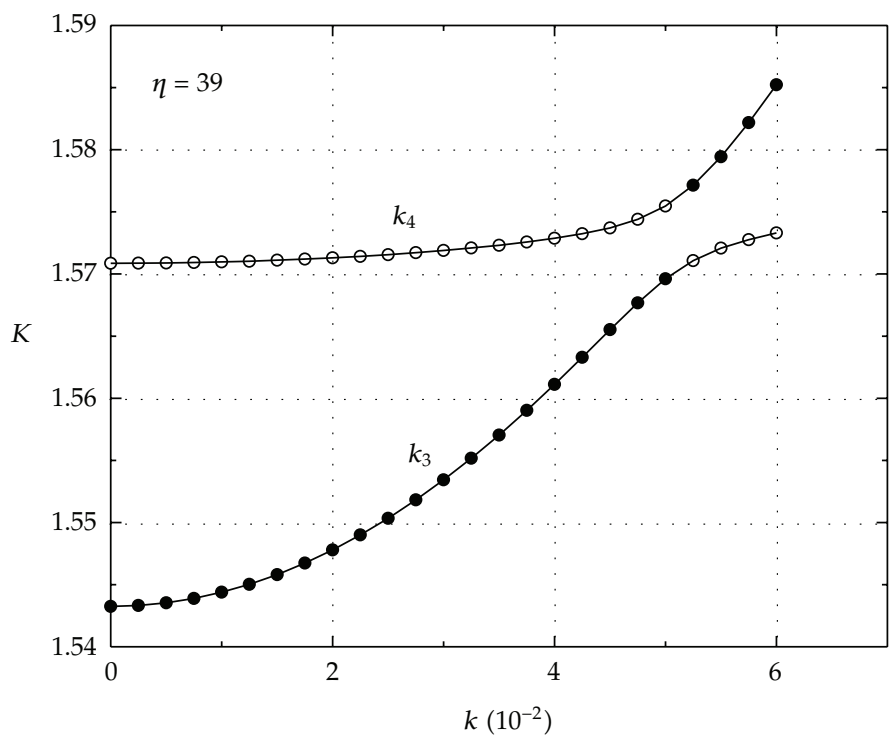

- Mode 3

○ Mode 4

Figure 11: The third and fourth natural frequencies verse the dimensionless angular velocity $\left(\alpha=0^{\circ}, r=1\right.$, $\eta=39)$.

\section{Acknowledgment}

The research was sponsored by the National Science Council, ROC, Taiwan, under contract NSC 98-2221-E-009-099-MY2.

\section{References}

[1] M. J. Schilhansl, "Bending frequency of a rotating cantilever beam," Journal of Applied Mechanics, vol. 25, pp. 28-30, 1958.

[2] R. O. Stafford and V. Giurgiutiu, "Semi-analytic methods for rotating Timoshenko beams," International Journal of Mechanical Sciences, vol. 17, no. 11-12, pp. 719-727, 1975.

[3] A. Leissa, "Vibrational aspects of rotating turbomachinery blades," Applied Mechanics Reviews, vol. 34, no. 5, pp. 629-635, 1981.

[4] D. H. Hodges and M. J. Rutkowski, "Free-vibration analysis of rotating beams by a variable-order finite-element method," AIAA Journal, vol. 19, no. 11, pp. 1459-1466, 1981.

[5] A. D. Wright, C. E. Smith, R. W. Thresher, and J. L. C. Wang, "Vibration modes of centrifugally stiffened beams," Journal of Applied Mechanics, vol. 49, no. 1, pp. 197-202, 1982.

[6] K. B. Subrahmanyam and K. R. V. Kaza, "Non-linear flap-lag-extensional vibrations of rotating, pretwisted, preconed beams including Coriolis effects," International Journal of Mechanical Sciences, vol. 29, no. 1, pp. 29-43, 1987.

[7] T. Yokoyama, "Free vibration characteristics of rotating Timoshenko beams," International Journal of Mechanical Sciences, vol. 30, no. 10, pp. 743-755, 1988.

[8] M. R. M. da Silva, C. L. Zaretzky, and D. H. Hodges, "Effects of approximations on the static and dynamic response of a cantilever with a tip mass," International Journal of Solids and Structures, vol. 27, no. 5, pp. 565-583, 1991.

[9] S. Y. Lee and Y. H. Kuo, "Bending frequency of a rotating beam with an elastically restrained root," Journal of Applied Mechanics, vol. 58, no. 1, pp. 209-214, 1991. 
[10] H. P. Lee, "Vibration on an inclined rotating cantilever beam with tip mass," Journal of Vibration and Acoustics, vol. 115, pp. 241-245, 1993.

[11] S. Naguleswaran, "Lateral Vibration of a centrifugally tensioned uniform Euler-Bernoulli beam," Journal of Sound and Vibration, vol. 176, no. 5, pp. 613-624, 1994.

[12] M. R. M. Crespo Da Silva, "A comprehensive analysis of the dynamics of a helicopter rotor blade," International Journal of Solids and Structures, vol. 35, no. 7-8, pp. 619-635, 1998.

[13] H. H. Yoo and S. H. Shin, "Vibration analysis of rotating cantilever beams," Journal of Sound and Vibration, vol. 212, no. 5, pp. 807-828, 1998.

[14] A. Bazoune, Y. A. Khulief, N. G. Stephen, and M. A. Mohiuddin, "Dynamic response of spinning tapered Timoshenko beams using modal reduction," Finite Elements in Analysis and Design, vol. 37, no. 3, pp. 199-219, 2001.

[15] S. C. Lin and K. M. Hsiao, "Vibration analysis of a rotating Timoshenko beam," Journal of Sound and Vibration, vol. 240, no. 2, pp. 303-322, 2001.

[16] P. Marugabandhu and J. H. Griffin, "A reduced-order model for evaluating the effect of rotational speed on the natural frequencies and mode shapes of blades," Journal of Engineering for Gas Turbines and Power, vol. 125, no. 3, pp. 772-776, 2003.

[17] J. R. Banerjee and H. Su, "Development of a dynamic stiffness matrix for free vibration analysis of spinning beams," Computers and Structures, vol. 82, no. 23-26, pp. 2189-2197, 2004.

[18] G. Wang and N. M. Wereley, "Free vibration analysis of rotating blades with uniform tapers," AIAA Journal, vol. 42, no. 12, pp. 2429-2437, 2004.

[19] A. A. Al-Qaisia, "Non-linear dynamics of a rotating beam clamped with an attachment angle and carrying an inertia element," Arabian Journal for Science and Engineering, vol. 29, no. 1, pp. 81-98, 2004.

[20] M. Sabuncu and K. Evran, "The dynamic stability of a rotating asymmetric cross-section Timoshenko beam subjected to lateral parametric excitation," Finite Elements in Analysis and Design, vol. 42, no. 5, pp. 454-469, 2006.

[21] S. Y. Lee and J. J. Sheu, "Free vibrations of a rotating inclined beam," Journal of Applied Mechanics, vol. 74, no. 3, pp. 406-414, 2007.

[22] S. Y. Lee and J. J. Sheu, "Free vibration of an extensible rotating inclined Timoshenko beam," Journal of Sound and Vibration, vol. 304, no. 3-5, pp. 606-624, 2007.

[23] W. R. Chen, "On the vibration and stability of spinning axially loaded pre-twisted Timoshenko beams," Finite Elements in Analysis and Design, vol. 46, no. 11, pp. 1037-1047, 2010.

[24] C. L. Huang, W. Y. Lin, and K. M. Hsiao, "Free vibration analysis of rotating Euler beams at high angular velocity," Computers and Structures, vol. 88, no. 17-18, pp. 991-1001, 2010.

[25] B. Yardimoglu, "A novel finite element model for vibration analysis of rotating tapered Timoshenko beam of equal strength," Finite Elements in Analysis and Design, vol. 46, no. 10, pp. 838-842, 2010.

[26] P. W. Likins, "Mathematical modeling of spinning elastic bodies for modal analysis," AIAA Journal, vol. 11, no. 9, pp. 1251-1258, 1973.

[27] J. C. Simo and L. Vu-Quoc, "The role of non-linear theories in transient dynamic analysis of flexible structures," Journal of Sound and Vibration, vol. 119, no. 3, pp. 487-508, 1987.

[28] K. M. Hsiao, "Corotational total Lagrangian formulation for three-dimensional beam element," AIAA journal, vol. 30, no. 3, pp. 797-804, 1992.

[29] K. M. Hsiao, R. T. Yang, and A. C. Lee, "Consistent finite element formulation for non-linear dynamic analysis of planar beam," International Journal for Numerical Methods in Engineering, vol. 37, no. 1, pp. 75-89, 1994.

[30] A. A. Shabana, H. A. Hussien, and J. L. Escalona, "Application of the absolute nodal coordinate formulation to large rotation and large deformation problems," Journal of Mechanical Design, vol. 120, no. 2, pp. 188-195, 1998.

[31] J. L. Escalona, H. A. Hussien, and A. A. Shabana, "Application of the absolute nodal co-ordinate formulation to multibody system dynamics," Journal of Sound and Vibration, vol. 214, no. 5, pp. 833850, 1998.

[32] T. J. Chung, Continuum Mechanics, Prentice Hall, Englewood Cliffs, NJ, USA, 1988.

[33] D. J. Malvern, Introduction to the Mechanics of the Continuous Medium, Prentice Hall, Englewood Cliffs, NJ, USA, 1969.

[34] S. S. Rao, Mechanical Vibrations, Addison-Wesley, 1986. 


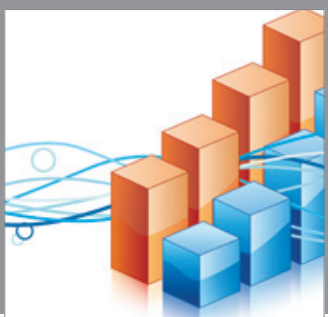

Advances in

Operations Research

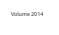

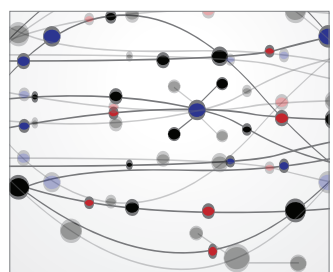

\section{The Scientific} World Journal
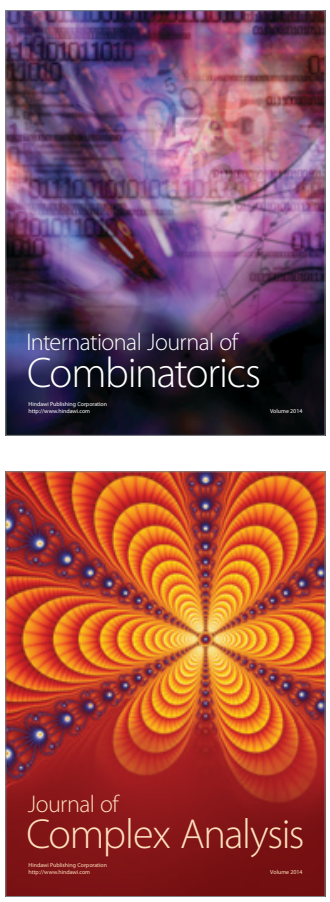

International Journal of

Mathematics and

Mathematical

Sciences
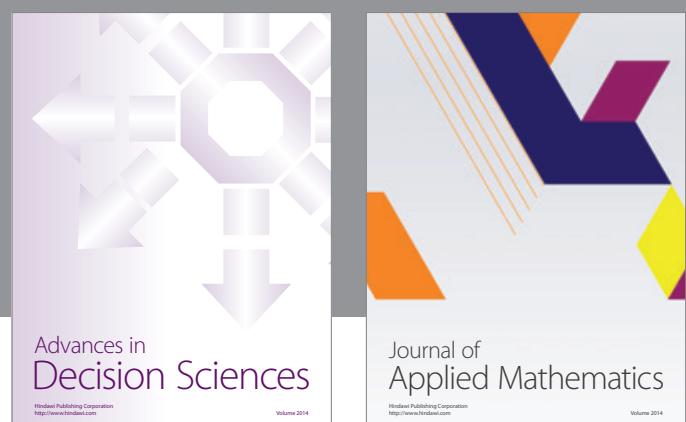

Journal of

Applied Mathematics
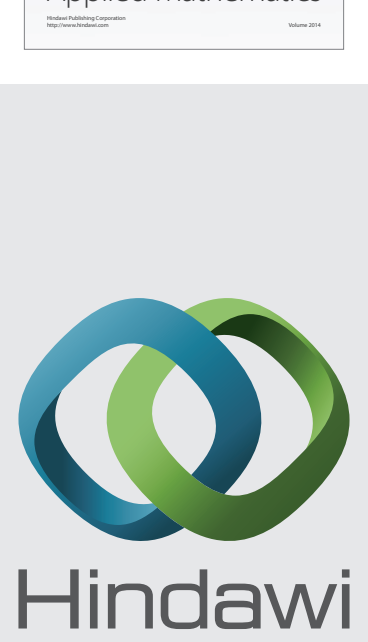

Submit your manuscripts at http://www.hindawi.com
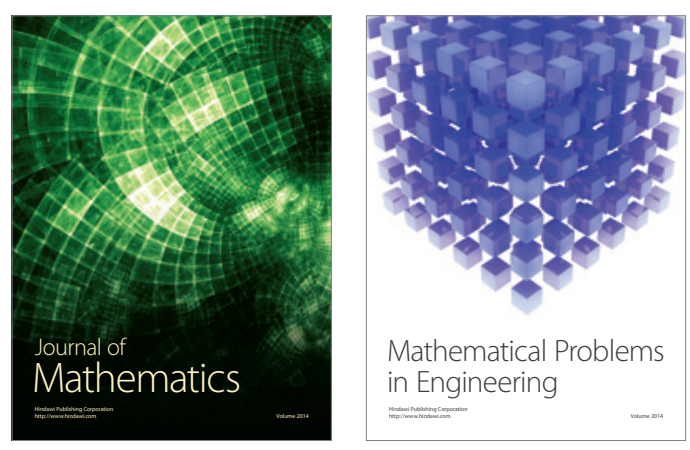

Mathematical Problems in Engineering
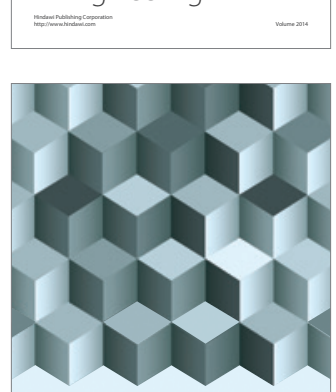

Journal of

Function Spaces
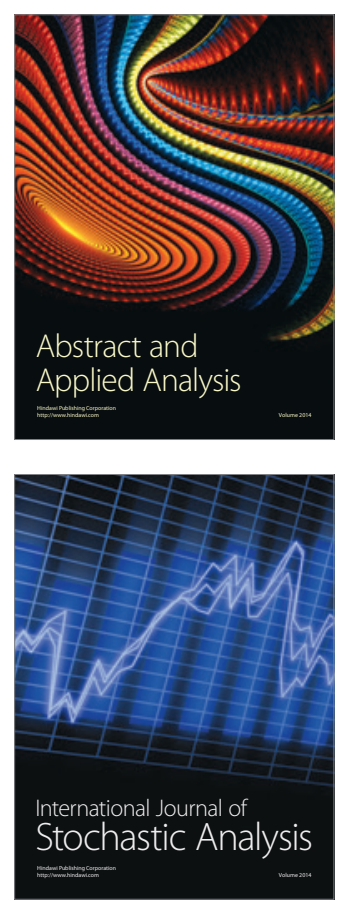

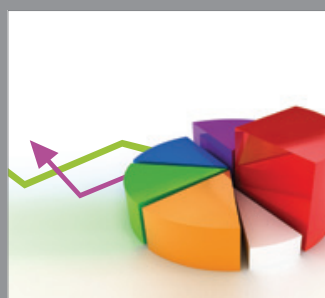

ournal of

Probability and Statistics

Promensencen
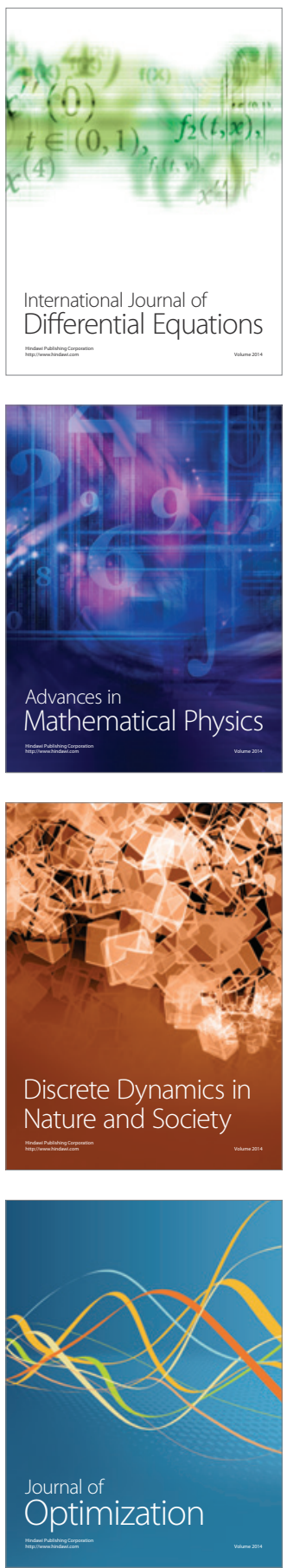